\title{
Atmospheric Variability Driven by Radiative Cloud Feedback in Brown Dwarfs and Directly Imaged Extrasolar Giant Planets
}

\author{
Xianyu Tan (10) and Adam P. Showman \\ Lunar and Planetary Laboratory, University of Arizona, 1629 University Boulevard, Tucson, AZ 85721, USA; xianyut@lpl.arizona.edu \\ Received 2018 June 6; revised 2019 February 26; accepted 2019 March 1; published 2019 March 28
}

\begin{abstract}
Growing observational evidence has suggested active meteorology in the atmospheres of brown dwarfs (BDs) and directly imaged extrasolar giant planets (EGPs). In particular, a number of surveys have shown that near-infrared brightness variability is common among $\mathrm{L}$ and $\mathrm{T}$ dwarfs. Despite the likelihood from previous studies that atmospheric dynamics is the major cause of the variability, the detailed mechanism of the variability remains elusive, and we need to seek a natural, self-consistent mechanism. Clouds are important in shaping the thermal structure and spectral properties of these atmospheres via their opacity, and we expect the same for inducing atmospheric variability. In this work, using a time-dependent one-dimensional model that incorporates a selfconsistent coupling between the thermal structure, convective mixing, cloud radiative heating/cooling, and condensation/evaporation of clouds, we show that radiative cloud feedback can drive spontaneous atmospheric variability in both temperature and cloud structure under conditions appropriate for BDs and directly imaged EGPs. The typical periods of variability are 1 to tens of hr, with a typical amplitude of the variability up to hundreds of $\mathrm{K}$ in effective temperature. The existence of variability is robust over a wide range of parameter space, but the detailed evolution of the variability is sensitive to model parameters. Our novel, self-consistent mechanism has important implications for the observed flux variability of BDs and directly imaged EGPs, especially for objects whose variability evolves on short timescales. It is also a promising mechanism for cloud breaking, which has been proposed to explain the $\mathrm{L} / \mathrm{T}$ transition of BDs.
\end{abstract}

Key words: brown dwarfs - methods: numerical - planets and satellites: atmospheres - planets and satellites: gaseous planets - radiative transfer

\section{Introduction}

Light-curve variability at infrared (IR) wavelengths is common among brown dwarfs (BDs) over a wide range of spectral types (e.g., Bailer-Jones \& Mundt 2001; Gelino et al. 2002; Clarke et al. 2008; Buenzli et al. 2014; Radigan et al. 2014; Wilson et al. 2014; Metchev et al. 2015; Leggett et al. 2016; Yang et al. 2016; Miles-Páez et al. 2017; see a recent review by Biller 2017 and Artigau 2018), as well as a handful of directly imaged extrasolar giant planets (EGPs; Biller et al. 2015, 2018; Zhou et al. 2016). The variability is thought to be caused by rotational modulation of inhomogeneous surface brightness (e.g., Radigan et al. 2012; Apai et al. 2013; Karalidi et al. 2016), which is partly supported by two-dimensional surface maps of a nearby BD (Crossfield et al. 2014). Shapes of light curves often evolve over a timescale of a few rotation periods (e.g., Artigau et al. 2009; Radigan et al. 2012; Biller et al. 2013; Gillon et al. 2013; Metchev et al. 2015; Apai et al. 2017), suggesting a rapid change of the surface features. Multiwavelength observations display pressure-dependent shifts of light-curve shapes for BDs of certain spectral types, indicating complex vertical structures for the surface variations (Buenzli et al. 2012; Apai et al. 2013; Yang et al. 2016).

The inhomogeneous brightness likely results from surface patchiness comprised of horizontally varying cloud and temperature structure (e.g., Radigan et al. 2012; Apai et al. 2013; Buenzli et al. 2015; Karalidi et al. 2016), but the mechanisms driving the surface patchiness and controlling its evolution remain elusive. Since clouds are tracers advected by atmospheric flows, and the clouds themselves result from dynamics, the patchiness also likely has a dynamical origin.
Only a few studies of atmospheric dynamics have been conducted under conditions appropriate for BDs and directly imaged EGPs. Local hydrodynamics simulations incorporating clouds by Freytag et al. (2010) and Allard et al. (2012) showed that gravity waves generated by interactions between the convective interior and the stratified layer can cause mixing that leads to small-scale cloud patchiness. However, dynamics in these local-scale models differs substantially from that at global scales. Moreover, surface patchiness at such local scales cannot by itself cause light-curve variability unless it is also accompanied by patchiness on much larger (regional-to-global) scales, and such larger-scale patchiness would necessarily be governed by very different dynamical processes. Using dynamical equations relevant for global scales, Showman \& Kaspi (2013) proposed that convectively excited waves could drive a global-scale circulation in the stratified atmosphere and generate horizontal patchiness in both temperature and cloud coverage, and they presented an analytic theory of how this process could work. Zhang \& Showman (2014) performed numerical simulations of such a global circulation using an idealized one-layer shallow-water model, with the goal of determining whether the atmospheric circulation resulting from such convective perturbations would exhibit zonal banding or patchy, isotropic turbulence. Nevertheless, three-dimensional (3D) models of this process are in their infancy. Circulation driven by latent heating associated with condensation of silicate clouds is able to generate large-scale cloud patchiness (Tan \& Showman 2017), but latent heating alone is rather weak to be considered as a dominant driver, given that horizontal temperature differences could be large in those highly 
variable BDs (e.g., Karalidi et al. 2016). Using a general circulation model coupled with parameterized thermal perturbations resulting from interactions between the convective interior and the stratified atmosphere, Showman et al. (2018b) showed that under conditions of relatively strong forcing and weak damping, robust zonal jets and the associated meridional circulation and temperature structure are common outcomes of the dynamics. They also demonstrated that long-term (multiple months to years) quasiperiodic oscillations of the equatorial zonal jets, similar to the quasi-biennial oscillation observed in Earth's stratosphere, can be driven by the thermal perturbations.

To date, no global dynamical model of BDs explicitly implements clouds and their radiative feedback to atmospheric flows. Opacities of clouds have a profound impact on the thermal structure, spectral properties, and thermal evolution of BDs (see reviews by Helling \& Casewell 2014 and Marley \& Robinson 2015). This should also be true in driving a vigorous global circulation and variability in these atmospheres, as the following reasoning suggests. Radiativeconvective equilibrium one-dimensional (1D) models predict very different temperature-pressure $(T-P)$ structures for models with roughly the same internal entropy but different cloud structures (see examples in, e.g., Tsuji 2002; Burrows et al. 2006). This could imply that different regions of the atmosphere (e.g., cloudy and relatively cloud-free regions) exhibit significantly different $T-P$ profiles, and the resulting horizontal temperature differences-as well as convective forcing from below-could then generate a 3D atmospheric circulation that helps to control and modulate the cloud patchiness. In addition, however, local processes within a single column (representing a regional patch on a BD) can potentially control whether that local patch is cloudy and lead to variability in that cloud patch's cloud structure over time. Because clouds are subjected to gravitational sedimentation and convective mixing, the latter being determined by the thermal structure, the changes of thermal structure should feed back onto clouds by changing the convective mixing, potentially maintaining variability by nonlinear self-interactions within the system. These two effects-large-scale cloud patchiness modulated by a $3 \mathrm{D}$ circulation and that resulting from convective mixing and cloud radiative processes within a single atmospheric column-can operate simultaneously in an atmosphere and interact with each other in a highly complex manner. Although one must eventually perform global dynamical models to truly decipher the circulation driven by these effects, such models would be extremely difficult to understand and diagnose. To build a systematic and clear understanding of the role of clouds in driving dynamics and variability, it is crucial to study the radiative cloud feedback starting from a 1D context where the effects can be cleanly isolated, as we do here.

In this study, we construct a simple time-dependent 1D atmospheric model that discards the equilibrium assumption to demonstrate the importance of radiative cloud feedback in driving the short-term atmospheric evolution. The time dependency is crucial because the radiative cloud feedback is essentially a nonsteady process. The model incorporates a self-consistent coupling between the thermal structure, convective mixing of tracers (including entropy, condensable vapor, and cloud particles), cloud radiative heating/cooling, and condensation/evaporation of clouds. The model is simple in the sense that each physical component is idealized but essential physical behaviors are preserved. The purposely simplified model allows us to better understand and diagnose the underlying mechanisms, as well as providing a much faster computational speed to explore the mechanism over a wide range of parameter space.

This paper is organized as follows. We introduce the numerical implementation in Section 2. We then present results, diagnoses of mechanisms, and sensitivity studies in Section 3. Finally, we discuss the implications in Section 4 and conclude in Section 5.

\section{Model}

We model the short-term evolution of a local atmospheric column of a BD or directly imaged EGP using a timedependent 1D model, which includes simple treatments for radiative transfer, condensation clouds, cloud gravitational settling, and convective mixing. We make several assumptions. First, cloud formation is determined by chemical equilibrium, which in our simple context implies condensation obeying a Clausius-Clapeyron-type relation. Second, we assume that the temperature at the model's bottom boundary remains fixed during the evolution, mimicking an atmosphere attached to a convective interior whose specific entropy does not evolve over short timescales. Third, vertical transport of heat and tracers by convection is modeled as a diffusion process, and the diffusion coefficient is determined by the local temperature lapse rate as a function of pressure and time. Fourth, we neglect the effects of condensation on gas thermodynamic properties, including the release of latent heat, and the effect of the condensates on the mean molecular weight and heat capacity. Finally, we neglect the advection terms. In principle, explicit vertical advection can be imposed as ascent or descent motion in 1D models. For a clear environment to understand the role of convective mixing (modeled as diffusion), we do not include explicit vertical advection in this study. We are interested in models where clouds first condense in the convective region.

Equations for temperature $T$, mixing ratio of condensable vapor $q_{v}$ (mass ratio between condensable vapor to the background dry $\mathrm{H}_{2}+\mathrm{He}$ gas, in units of $\mathrm{kg} \mathrm{kg}^{-1}$ ), and mixing ratio of condensates $q_{c}\left(\mathrm{~kg} \mathrm{~kg}^{-1}\right)$ as a function of height $z$ and time $t$ are as follows (a similar set of time-dependent 1D equations can be found in Smith \& Gierasch 1995):

$$
\begin{gathered}
\frac{d T}{d t}=-\frac{1}{c_{p} \rho} \frac{\partial F}{\partial z}+\frac{1}{c_{p} \rho} \frac{\partial}{\partial z}\left(c_{p} \rho K_{\mathrm{zz}}\left[\frac{\partial T}{\partial z}-\left(\frac{\partial T}{\partial z}\right)_{\mathrm{ad}}\right]\right) \\
\frac{d q_{v}}{d t}=-\frac{q_{v}-q_{s}}{\tau_{c}} \delta+\frac{\min \left(q_{s}-q_{v}, q_{c}\right)}{\tau_{c}}(1-\delta) \\
+\frac{1}{\rho} \frac{\partial}{\partial z}\left(K_{\mathrm{zz}} \rho \frac{\partial q_{v}}{\partial z}\right)+Q_{\text {deep }}
\end{gathered}
$$




$$
\begin{aligned}
\frac{d q_{c}}{d t}= & \frac{q_{v}-q_{s}}{\tau_{c}} \delta-\frac{\min \left(q_{s}-q_{v}, q_{c}\right)}{\tau_{c}}(1-\delta) \\
& +\frac{1}{\rho} \frac{\partial\left(\rho\left\langle q_{c} V_{s}\right\rangle\right)}{\partial z}+\frac{1}{\rho} \frac{\partial}{\partial z}\left(K_{\mathrm{zz}} \rho \frac{\partial q_{c}}{\partial z}\right),
\end{aligned}
$$

where $(\partial T / \partial z)_{\mathrm{ad}}$ is the adiabatic temperature lapse rate; $q_{s}$ is the local saturation vapor mixing ratio determined by a given condensation function, local temperature, and pressure; $V_{s}$ is the settling speed of particles; $K_{\mathrm{zz}}$ is the time- and pressuredependent vertical diffusion coefficients in height coordinates; $\rho$ is the gas density; $\tau_{c}$ is the conversion timescale representing the source/sink of tracers due to condensation or evaporation; $c_{p}$ is the specific heat at constant pressure; $g$ is the surface gravity; and $F$ is the net radiative flux. The term $Q_{\text {deep }}=-\left(q_{v}-q_{\text {deep }}\right) / \tau_{\text {deep }}$ applies only at pressure regions deeper than 50 bars, relaxing local vapor $q_{v}$ to the deep mixing ratio $q_{\text {deep }}$ over a characteristic timescale $\tau_{\text {deep }}$, which is generally set to $10^{3} \mathrm{~s}$, consistent with mixing timescales over a pressure scale height near the lower model boundary. Moderate deviation of $\tau_{\text {deep }}$ from this value does not affect the model results. We have performed additional experiments with $\tau_{\text {deep }}=10^{2}$ and $10^{4} \mathrm{~s}$, and the variability in these sensitivity tests is almost identical to that with $\tau_{\text {deep }}=10^{3} \mathrm{~s}$.

The first two terms on the right-hand side of Equations (2) and (3) are sources/sinks due to condensation and evaporation, respectively, where $\delta=1$ if vapor $q_{v}$ is supersaturated and $\delta=0$ otherwise. ${ }^{1}$ The conversion timescale $\tau_{c}$ is assumed to be very short ( $10 \mathrm{~s}$; e.g., Helling \& Casewell 2014) compared to the cloud settling or thermal evolution timescales. We will discuss the sensitivity to different conversion timescales. The particle settling velocity in the third term on the right-hand side of Equation (3) is calculated following Equations (3)-(7) in Parmentier et al. (2013), ${ }^{2}$ and the bracket \langle\rangle represents the settling flux integrated over the particle size distribution. We implement enstatite $\left(\mathrm{MgSiO}_{3}\right)$ to represent the silicate cloud, which is one of the most abundant condensates in $\mathrm{L}$ and $\mathrm{T}$ dwarfs. Assuming solar abundance, the expression for the total gas pressure $P_{\mathrm{T}}$ (in unit of bars) at which enstatite saturates as a function of temperature is taken from Visscher et al. (2010):

$$
10^{4} / T=6.26-0.35 \log P_{T}
$$

To obtain the familiar Clausius-Clapeyron-type expression for the local vapor saturation mixing ratio $q_{s}$, we simply use $q_{s}=P_{T} q_{\text {deep }} / p$, which comes with assuming all silicate vapor

\footnotetext{
1 The expression $\frac{\min \left(q_{s}-q_{v}, q_{c}\right)}{\tau_{c}}$ for evaporation is a compact form of

$$
\begin{cases}\frac{q_{s}-q_{v}}{\tau_{c}} & q_{c} \geqslant q_{s}-q_{v} \\ \frac{q_{c}}{\tau_{c}} & q_{c}<q_{s}-q_{v},\end{cases}
$$

in which the first condition implies that the vapor field is relaxed toward saturation when the number of cloud particles is sufficient for conversion, whereas the second condition refers to the fact that only the existing number of cloud particles can be converted to vapor when the particles are insufficient.

2 The expression of the terminal fall velocity of a spherical particle can be found in Pruppacher \& Klett (2012). For detailed references of the settling velocity in the context of gas giants, readers are referred to the references in Parmentier et al. (2013) or Ackerman \& Marley (2001).
}

condense into enstatite. The deep mixing ratio $q_{\text {deep }}$ is about 0.0026 using the molar fraction of $\mathrm{Mg}$ relative to $\mathrm{H}_{2}+\mathrm{He}$ in solar abundance from Lodders (2003) and assuming all silicate vapor condense into enstatite.

In our numerical implementation, Equations (1)-(3) are solved in pressure $p$ coordinates, which can be converted from height $z$ coordinates using the hydrostatic assumption $\partial p / \partial z=-\rho g$. We integrate the system forward with time using the third-order Adams-Bashforth scheme (Durran 1991), which has been frequently used in atmospheric dynamical models. The value of a prognostic variable, $T$ for example, at time $t+\Delta t$ is marched forward by

$$
\begin{aligned}
T(t+\Delta t)= & T(t)+\frac{\Delta t}{12}\left[23 \frac{d T}{d t}(t)\right. \\
& \left.-16 \frac{d T}{d t}(t-\Delta t)+5 \frac{d T}{d t}(t-2 \Delta t)\right] .
\end{aligned}
$$

At the initialization, we first integrate the system two time steps using a simple Euler scheme, then switch to the thirdorder Adam-Bashforth scheme. The time step is chosen to be small enough $(0.1 \mathrm{~s}$ for simulations with nominal vertical resolution) to guarantee stability and convergence. Our initial condition includes a cloud-free radiative-convective equilibrium profile, solar abundance of silicate vapor below condensation level, and no clouds. After sufficient integration time, the system reaches a statistical equilibrium state that is insensitive to initial conditions. ${ }^{3}$ The computation domain ranges from $10^{-3}$ to 100 bars that are discretized into 100 layers. We have tested additional models with different vertical resolutions of 50, 200, and 300 layers (shown in Appendix A). The results show good agreement for a resolution of 100 layers or more. For computational efficiency, we use 100 layers for models presented in this work. We emphasis that our model does not implement external ad hoc forcing like those in Zhang \& Showman (2014) and Robinson \& Marley (2014), and the atmospheric activity shown below arises spontaneously from self-interactions of the system.

\subsection{Cloud Size Distribution}

We assume a constant cloud particle number per dry air mass $\mathcal{N}_{c}$ (in unit of $\mathrm{kg}^{-1}$ ) throughout the atmospheric column, then use this number to determine local cloud propertiessuch as the time-varying, pressure-dependent mean particle size-given the time- and pressure-dependent amount of condensate. In our nominal models, we prescribe a lognormal particle size distribution, which has been widely used to parameterize clouds in BD atmospheres (e.g., Ackerman \& Marley 2001; Barman et al. 2011; Morley et al. 2012):

$$
n(r)=\frac{\mathcal{N}_{c}}{\sqrt{2 \pi} \sigma r} \exp \left(-\frac{\left[\ln \left(r / r_{0}\right)\right]^{2}}{2 \sigma^{2}}\right),
$$

\footnotetext{
The statistical equilibrium is not steady. As will be shown in the next section, there are large temporal fluctuations around what amounts to a mean "climate." We have tested the models with vastly different initial conditions. When reaching statistical equilibrium, the mean climate of the systemincluding the amplitude and period of the variability-is independent of the initial conditions.
} 
where $r$ is the particle radius, $n(r)=d \mathcal{N}_{c} / d r$ is the number density distribution, $r_{0}$ is a reference radius, and $\sigma$ is a nondimensionalized constant that measures the width of the distribution. The number density of particles peaks at a radius slightly less than $r_{0}$ and exponentially decreases with radius for radii smaller or larger than the radius corresponding to this peak. This function aims at capturing a possibly broad spread of particle size distribution (see a detailed discussion in Ackerman $\&$ Marley 2001). However, the parameter $\sigma$ controlling the width of the size distribution is unconstrained. Here for the nominal models, we empirically assume $\sigma=1$ and will test the sensitivity to varying $\sigma$. With the cloud mass-mixing ratio $q_{c}$ obtained from Equation (3), and given the specified values of $\sigma$ and $\mathcal{N}_{c}$, the particle size distribution can be determined by calculating the reference radius $r_{0}$. This is done by solving the integral $q_{c}=\frac{4}{3} \pi \rho_{c} \int_{0}^{\infty} r^{3} n(r) d r=\frac{4}{3} \pi \rho_{c} \mathcal{N}_{c} r_{0}^{3} \exp \left(\frac{9}{2} \sigma^{2}\right)$, which is simply the definition of the cloud mass-mixing ratio. Practically, when summing over the particle size distribution for calculation of cloud opacity and gravitational settling flux, we discard particles smaller than a minimal radius $r_{\min }$ and a maximum radius $r_{\max }$, the latter of which is set to $100 \mu \mathrm{m}$. We choose $0.01 \mu \mathrm{m}$ for $r_{\text {min }}$ following Tsuji (2002), who estimated that in typical BD conditions, $0.01 \mu \mathrm{m}$ is roughly the criterion below which the cloud particle may not be stable against surface tension. In fact, our numerical results are not sensitive to the choice of $r_{\min }$ as long as it is sufficiently small, because opacity contributions from particles smaller than $\sim 0.01 \mu \mathrm{m}$ are negligible in determining the heating/cooling rates that drive the evolution.

We have also implemented an exponential particle size distribution that has been widely used to describe precipitation in Earth's atmosphere (e.g., Chapter 2, Straka 2009),

$$
n(r)=\frac{\mathcal{N}_{c}}{r_{0}} \exp \left(-\frac{r}{r_{0}}\right)
$$

to test the sensitivity to different assumed size distributions. The detailed evolution of atmospheric variability differs with different size-distribution function, but the mechanism and qualitative behavior remain the same. For conciseness, we will not present the results of the exponential size distribution in the main text. However, Appendix C presents some tests demonstrating that models with both size-distribution functions exhibit qualitatively similar behavior.

\subsection{Radiative Transfer}

We model the atmospheric radiative transfer using a planeparallel, two-stream approximation. We focus on a gray atmosphere with a single broad thermal band for simplicity and computational efficiency. The radiative transfer equations in an absorbing, emitting, and multiple-scattering atmosphere with the $\delta$-function adjustment for scattering are solved using the efficient numeral package TWOSTR (Kylling et al. 1995, wherein the radiative equations solved in our model can be found). Komacek et al. (2017) presented tests quantifying the model behavior relative to analytically known solutions for simple cases; these tests demonstrate that our numerical implementation agrees extremely well with known analytic solutions. The background model atmosphere uses a frequencyaveraged gas opacity, the Rosseland-mean opacity $\kappa_{\mathrm{R}, \mathrm{g}}$, in all pressures from Freedman et al. (2014) with solar composition. Since we are mostly interested in the cloud condensation region, where it is optically thick, the Rosseland-mean opacity gives a good estimation of radiation flux in this limit. In the upper atmosphere, where it is optically thin, there is no good choice a priori for a single opacity in the gray approximation.

Cloud particles interact with radiation via absorption and scattering, which are parameterized by the extinction coefficient $Q_{\text {ext }}$, scattering coefficient $Q_{\text {scat }}$, and asymmetry parameter $\tilde{g}$. To be consistent with the background Rosseland-mean gaseous opacity, the total cloud extinction opacity $\kappa_{\mathrm{R} \text {,ext }}$ is averaged over wavelength using the Rosseland-mean definition,

$$
\frac{1}{\kappa_{\mathrm{R}, \mathrm{ext}}}=\frac{\int_{0}^{\infty} \frac{1}{\kappa_{\mathrm{ext}}(\lambda)} \frac{d B_{\lambda}}{d T} d \lambda}{\int_{0}^{\infty} \frac{d B_{\lambda}}{d T} d \lambda},
$$

where $B_{\lambda}$ is the Planck function and $\kappa_{\text {ext }}(\lambda)=$ $\int_{r_{\min }}^{\infty} n(r) \pi r^{2} Q_{\text {ext }}(r, \lambda) d r$ is the total cloud opacity at $\lambda$ summing over all particle sizes. The cloud scattering opacity $\kappa_{\mathrm{R} \text {,scat }}$ and $\tilde{g}$ are defined likewise. Assuming spherical particles, the coefficients $Q_{\text {ext }}(r, \lambda), Q_{\text {scat }}(r, \lambda)$, and $\tilde{g}(r, \lambda)$ are precalculated with Mie theory using the numerical package written by Schäfer et al. (2012) with the refractive index of enstatite obtained from Jäger et al. (2003). The total opacity is simply the sum of gas and cloud opacity $\kappa=\kappa_{\mathrm{R}, \mathrm{g}}+\kappa_{\mathrm{R} \text {,ext }}$. Note that although we adopt the specific optical properties of enstatite particles for our nominal models, our qualitative results remain robust over a wide range of cloud optical properties. In addition to our nominal models, we performed a range of simulations varying the scattering coefficient, extinction coefficient, and asymmetry parameter over plausible ranges; these simulations demonstrate overall behavior (including temporal variability) that qualitatively resembles that of our nominal models.

\subsection{Diffusion}

Convective mixing for entropy and tracers is modeled as diffusion processes. The time- and pressure-dependent vertical diffusion coefficient $K_{\mathrm{zz}}$ in height coordinates depends on the temperature lapse rate. We adopt the classical expression of the diffusion coefficient based on the mixing-length theory (Gierasch \& Goody 1968),

$$
K_{\mathrm{zZ}}=\left\{\begin{array}{l}
0, \quad \frac{\partial \ln T}{\partial \ln p}-\left(\frac{\partial \ln T}{\partial \ln p}\right)_{\mathrm{ad}} \leqslant 0 \\
l^{2} \frac{g}{\sqrt{R T}} \sqrt{\frac{\partial \ln T}{\partial \ln p}-\left(\frac{\partial \ln T}{\partial \ln p}\right)_{\mathrm{ad}}}, \quad \text { else }
\end{array}\right.
$$

where $l$ is the mixing length, which we set equal to the local pressure scale height $H_{p}=\frac{R T}{g}$. By definition, the convective diffusion coefficient is only effective in the convective region where $\frac{\partial \ln T}{\partial \ln p}-\left(\frac{\partial \ln T}{\partial \ln p}\right)_{\mathrm{ad}}>0$, so outside the convective region, 

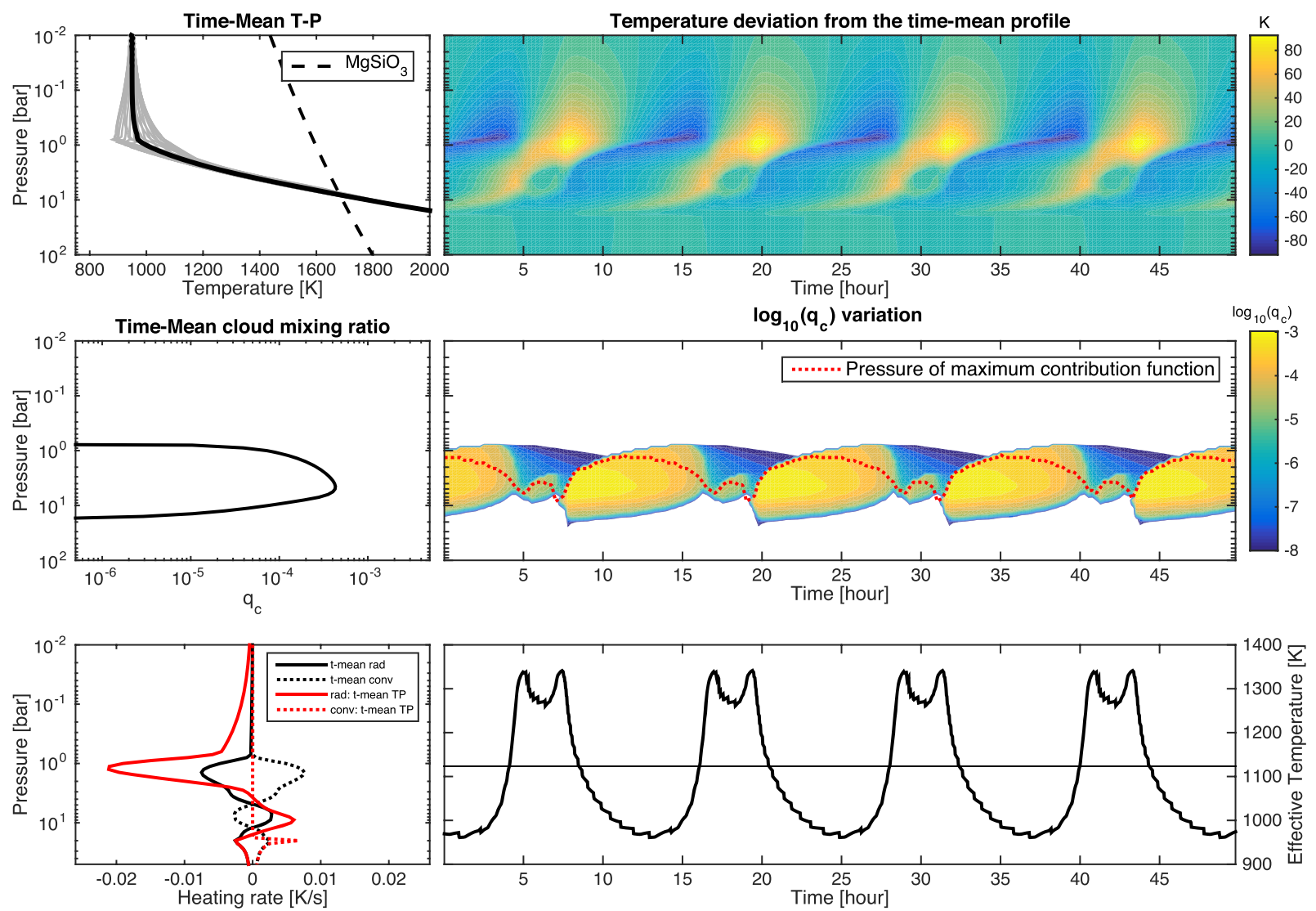

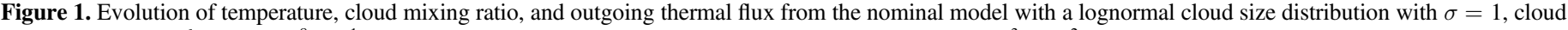

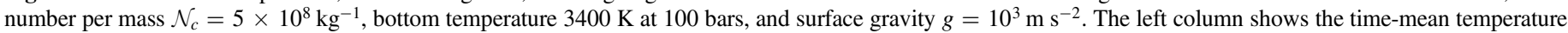

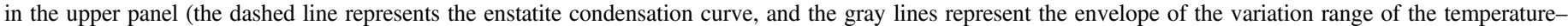

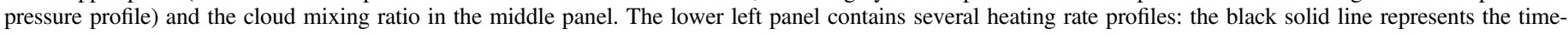

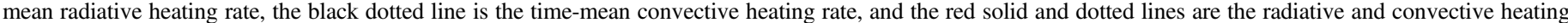

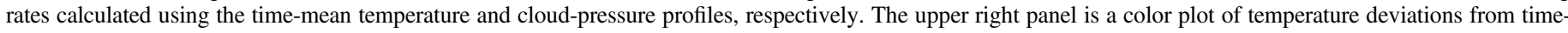

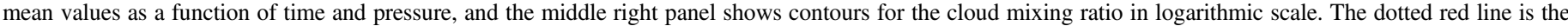

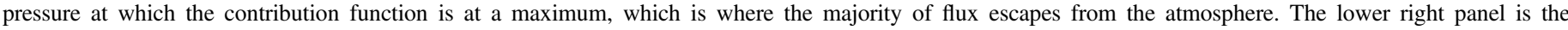

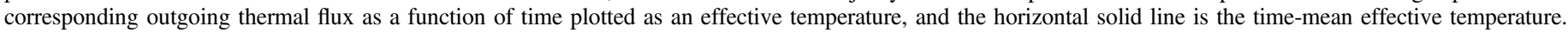

$K_{\mathrm{zz}}$ is set to zero in the nominal setup. However, small-scale eddies or large-scale flows could provide transport of tracers in the stratified regions. Because quantifying the mixing in the stratified atmosphere is difficult, as the atmospheric conditions are unknown, in some experiments, we impose a minimal value of $K_{\mathrm{zz}}$ as a background value to the diffusion coefficient for tracers (cloud and vapor) to crudely represent mixing in the stratified layers, and we will discuss the results in the sensitivity studies section.

\section{Results}

\subsection{A Nominal Case}

We start by describing a nominal model with a lognormal cloud size distribution with $\sigma=1$, cloud number per mass $\mathcal{N}_{c}=5 \times 10^{8} \mathrm{~kg}^{-1}$, bottom temperature $3400 \mathrm{~K}$ at 100 bars, and surface gravity $g=10^{3} \mathrm{~m} \mathrm{~s}^{-2}$. If there were no clouds, the equilibrium solution would have an effective temperature $\sim 1380 \mathrm{~K}$. The time-averaged $T-P$ profile is shown in the upper left panel of Figure 1, and the time-averaged cloud mixing ratio is shown in the middle left panel. The intersection between the condensation curve and the mean $T-P$ profile indicates that the predicted condensation level is at about 8 bars. The time-mean cloud layer extends vertically across more than two pressure scale heights. The cloud structure undergoes significant variation over short timescales, while the temperature structure varies moderately. The gray lines in the upper left panel of Figure 1 represent an envelope of $T-P$ variations, and the right column shows (from top to bottom) the temperature deviations relative to the time-averaged profile, the cloud mixing ratio as a function of time and pressure, and the outgoing thermal flux (expressed as an effective temperature), respectively, all as a function of time. The evolution of these quantities shows fairly regular oscillation patterns with a period of about $12 \mathrm{hr}$. The temperature variation on isobars reaches a maximum of about $180 \mathrm{~K}$ at around 0.8 bar and gradually decreases above and below this level. The cloud mixing ratio and layer thickness vary significantly during the evolution, ranging from a thick cloud layer that extends more than two pressure scale heights to an almost cloud-free atmosphere. The time-averaged outgoing thermal flux is $\sim 1125 \mathrm{~K}$ in terms of effective temperature, much lower than that of the cloud-free model; this difference results from the higher and thus cooler emission level associated with the cloud top. ${ }^{4}$ The amplitude of the effective temperature variation exceeds $350 \mathrm{~K}$, much larger than the actual temperature variation on isobars. The outgoing flux

\footnotetext{
4 Here the cloud top is loosely defined as the level below which the cloud opacity rapidly exceeds the background gaseous opacity.
} 
variation is mainly caused by altitude variation of the cloud top and thus the emission-level temperature variation, though the actual temperature variations (on isobars) also contribute positively. This is demonstrated by showing that the pressure level at which the contribution function reaches a maximum (the dotted line in the middle panel of Figure 1) closely follows the evolution of the cloud top.

The system is in a statistical equilibrium state, meaning that the time-averaged radiative flux divergence is balanced by convective flux divergence, and the tracer settling flux is balanced by convective mixing flux. This is illustrated in the lower left panel of Figure 1, where the time-averaged radiative and convective heating rates (the black solid and dotted lines, respectively) are equal in magnitude but of opposite sign. However, this equilibrium state cannot be characterized using the time-averaged $T-P$ and cloud profiles due to the variability and nonlinearity between temperature and flux. The radiative and convective heating rates of the time-averaged temperature and cloud profiles are also plotted in the lower left panel of Figure 1 as red lines. The significant nonzero net heating rate suggests that this single set of time-averaged profiles is not in equilibrium. In addition, the outgoing thermal flux of the time-averaged profiles is not equal to the timeaveraged outgoing thermal flux. This is always the case for models showing variability. This makes sense because the thermal and tracer profiles are imbalanced at any instant, and the time averages of them are therefore not necessarily in equilibrium. Our results suggest that a single set of $T-P$ profiles cannot represent a statistical equilibrium of an atmosphere with vigorous cloud formation.

There are small-scale jumps in the flux curve shown in Figure 1, which are caused by the rapid changes of the cloudtop pressure when a new convectively unstable layer formed above the cloud top. The period and amplitude of the jumps in flux depend on the numerical resolution of the pressure grid. As shown in our resolution test in Appendix A, higher resolution results in a smoother flux curve, but the overall quantitative variability remains almost the same. We emphasize that these jumps are not numerical errors but physical behaviors resulting from finite numerical resolution.

Next, we show various diagnostic quantities in an evolution cycle of the system, which helps to clarify the mechanism by which the variability is driven. Figure 2 contains the quantities at different time snapshots. The potential temperature in the first column is defined as $\theta=T\left(p_{0} / p\right)^{R / c_{p}}$, referring to the temperature an air parcel would have if it is adiabatically compressed or expensed to a reference pressure $p_{0}$, and $R$ is the specific gas constant. We choose $\theta$ instead of temperature to represent thermal structure because convective regions can be easily recognized as having a nearly constant $\theta$ with pressure. Tracer abundances, including the vapor mixing ratio $q_{v}$, saturated vapor mixing ratio $q_{v, s}$, and cloud mixing ratio $q_{c}$, are shown in the second column; the total gray thermal opacity is shown in the third column; the radiative heating/cooling rate is shown in the fourth column; and the diffusion coefficient $K_{\mathrm{zz}}$ is shown in the fifth column.

Comparisons between the potential temperature $\theta$, cloud mixing ratio $q_{c}$, and diffusion coefficient $K_{\mathrm{zz}}$ profiles suggest that the cloud layer always corresponds to a secondary detached convective layer. In other words, the formation of clouds generates a stratification below the cloud base where there would otherwise be a convective region. The detached convective zone associated with cloud formation has also been revealed by many studies (e.g., Tsuji 2002; Burrows et al. 2006). Large cloud opacity creates an opacity inversion near the cloud base (see the opacity structure in the third column), which leads to heating below the cloud base, stratifying the nearby layers. Meanwhile, strong radiative cooling occurs at the cloud top due to the sharp vertical opacity gradient caused by the sharp cloud top. The combination of cooling at the top and heating at the bottom maintains a convective instability within the cloud layer that is responsible for the secondary convective layer. In turn, the secondary convective layer provides strong mixing that can almost well mix the bulk particle mixing ratio within the layer, maintaining the cloud layer against rapid gravitational settling and creating a sharp cloud top. The mixing efficiency can be estimated by comparing two timescales: the diffusion timescale over a scale height $H_{p}^{2} / K_{\mathrm{zz}}$ and settling timescale $H_{p} / v_{\text {set }}$, where $v_{\text {set }}$ is the settling velocity in height coordinates (which can be found in, e.g., Equation (3) in Parmentier et al. 2013). For example, based on the model solution, the typical diffusion coefficient in the cloud layer is $\sim 2 \times 10^{5} \mathrm{~m}^{2} \mathrm{~s}^{-1}$; this value implies a diffusion timescale similar to the settling timescale for particles of $\sim 40 \mu \mathrm{m}$. This implies that particles smaller than $\sim 40 \mu \mathrm{m}$ can easily be lofted by convective mixing. In this particular simulation, the particle size distribution peaks at around $0.4 \mu \mathrm{m}$ when the cloud layer is thick, so the majority of the particle settling flux can be roughly balanced by the mixing within the cloud layer. Above the cloud top, the atmosphere is stratified; otherwise, convection would quickly mix clouds upward until reaching the stratified region. There, $K_{\mathrm{zz}}$ is zero or, for practical purposes, much smaller than the $K_{\mathrm{zz}}$ in the convective region, so it is unable to mix much cloud, therefore creating a sharp cloud top.

The results show a high supersaturation of condensable vapor in the secondary convective zone-the vapor abundance is much higher than the equilibrium saturation vapor abundance determined by Equation (5). This can be seen in the second column of Figure 2. With a finite conversion timescale $\tau_{c}$ between vapor and cloud, the high supersaturation is caused by the strong convective mixing. Because of the abundant vapor at altitudes slightly above the condensation level, the source of the clouds there is actually condensation, with a nearly balanced sink from convective mixing that distributes clouds toward higher altitudes and below the condensation level. To quantify the degree of supersaturation as a function of $\tau_{c}$ and $K_{\mathrm{zz}}$, one can compare the lapse rate of the equilibrium vapor mixing ratio $d \ln q_{s} / d \ln p$ (which is determined only by temperature) to that of the actual vapor mixing ratio $d \ln q_{v} / d \ln p$ (which is determined by both microphysical and mixing timescales). Using Equation (5), the relation $q_{s}=P_{T} q_{\text {deep }} / p$, and assuming a nearly adiabatic $T-P$ profile $\left(d \ln T / d \ln p \sim R / c_{p}\right)$, one can get $d \ln q_{s} / d \ln p=\frac{R}{c_{p}} \frac{65788}{T}-1$. The lapse rate of the actual vapor mixing ratio can be estimated by assuming a quasi-balance between condensation and convective mixing in the pressure-coordinate version of Equation (2): $\frac{q}{\tau_{c}} \sim \frac{d}{d p}\left(K_{\mathrm{zz}}(\rho g)^{2} \frac{d q_{v}}{d p}\right)$, where we assume $q_{s} \ll q$. Treating $K_{\mathrm{zz}}$ and $d \ln q_{v} / d \ln p$ as nearly constant on the local scale, we have $d \ln q_{v} / d \ln p \sim \sqrt{\frac{R^{2} T^{2}}{\tau_{c} K_{z z} g^{2}}+\frac{1}{4}}-\frac{1}{2}$. Thus, the ratio of 


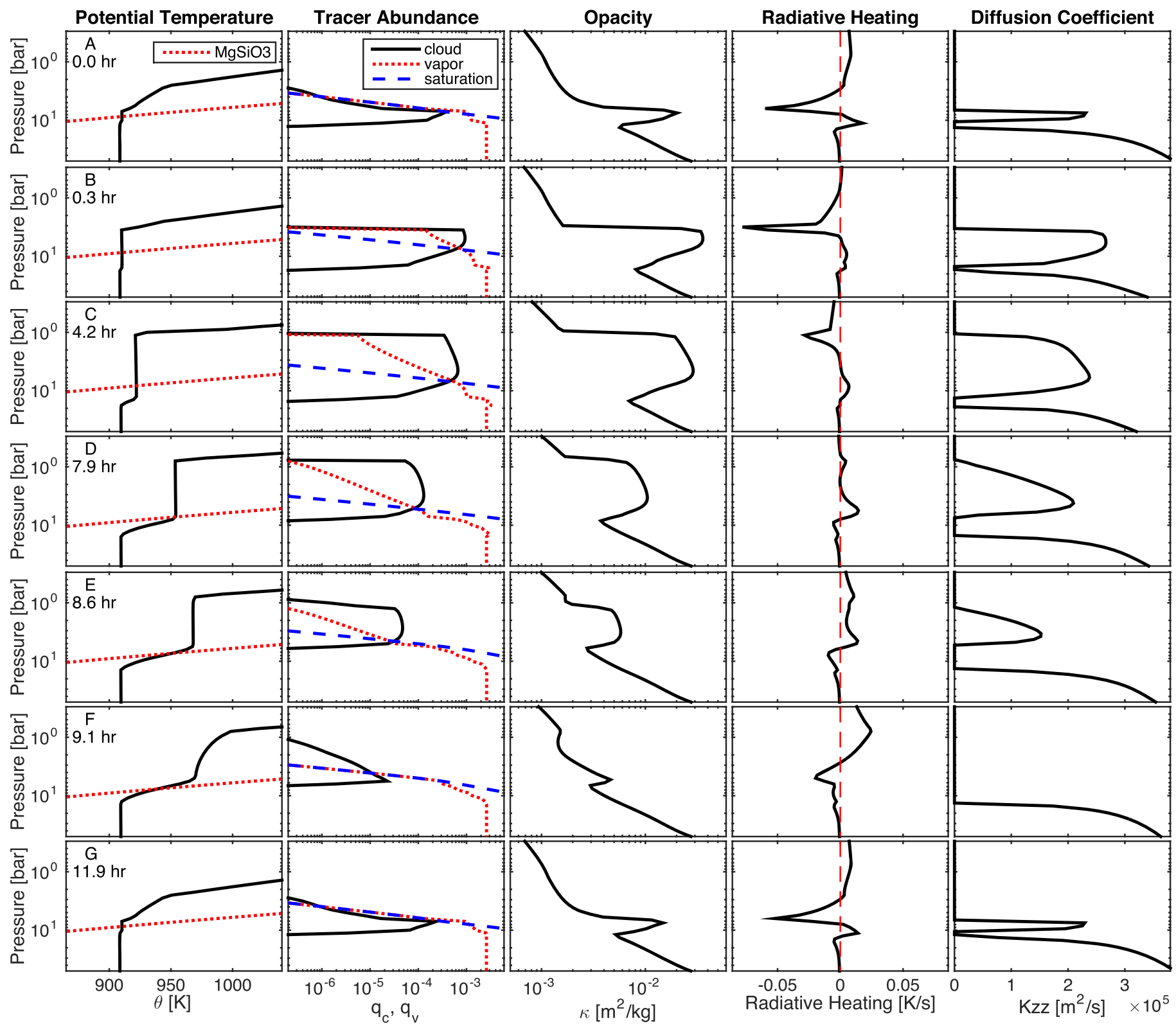

Figure 2. Here we show several diagnostic quantities that are used to illustrate the detailed evolution and mechanism of the variability. The sequence is shown from a complete cycle of the nominal case shown in Figure 1. Each row represents a snapshot of the atmospheric state at a given time, with time increasing downward as marked at the beginning of each row. The first column is the potential temperature $\theta$, and the dashed lines show the enstatite $\left(\mathrm{MgSiO}_{3}\right)$ condensation curve in $\theta_{-}$ pressure space. The second column contains the cloud mixing ratio $q_{c}$ (solid lines), vapor mixing ratio $q_{v}$ (dotted lines), and saturation vapor mixing ratio $q_{s}$ (dashed lines). The third column is the total opacity $\kappa$ and the fourth column is the radiative heating/cooling rate. Finally, the fifth column shows the vertical diffusion coefficient $K_{\mathrm{zz}}$.

lapse rates is

$$
\frac{d \ln q_{s}}{d \ln p} / \frac{d \ln q_{v}}{d \ln p} \sim \frac{\frac{R}{c_{p}} \frac{65788}{T}-1}{\sqrt{\frac{R^{2} T^{2}}{\tau_{c} K_{\mathrm{zz}} g^{2}}+\frac{1}{4}}-\frac{1}{2}} .
$$

Assuming $T \sim 1300 \mathrm{~K}, K_{\mathrm{zz}} \sim 2 \times 10^{5} \mathrm{~m}^{2} \mathrm{~s}^{-1}$, and $\tau_{c}=10 \mathrm{~s}$, one can get a ratio around 4.5, meaning that the supersaturation $q_{v} / q_{s}$ could reach the order of 100 only one pressure scale height above the condensation level, roughly consistent with the model results. For the same reason, some fraction of clouds can be mixed well below the condensation level, even with a very short evaporation timescale due to efficient convective mixing.
A few features are interesting. First, temperature variations exhibit a pressure-dependent shift, as seen from the tilted patterns in the upper right panel of Figure 1, and the maximum phase difference is between the cloud top and base. This shift is a result of the transition between convection and stratification in the cloud-forming region shown in Figure 2. Second, thick clouds usually correlate to a convective and cooler profile, and thin clouds correlate to a stratified and warmer profile. This is because the secondary convective layer and cloud layer are coupled and coevolve in time. When clouds are present, the atmospheric structure is forced to be nearly adiabatic, which is cool above the condensation level. When the cloud dissipates, the region where there were clouds is stratified and thus warmer. Finally, the instantaneous cloud-base pressure varies 


\section{Mechanism of cloud top growth}
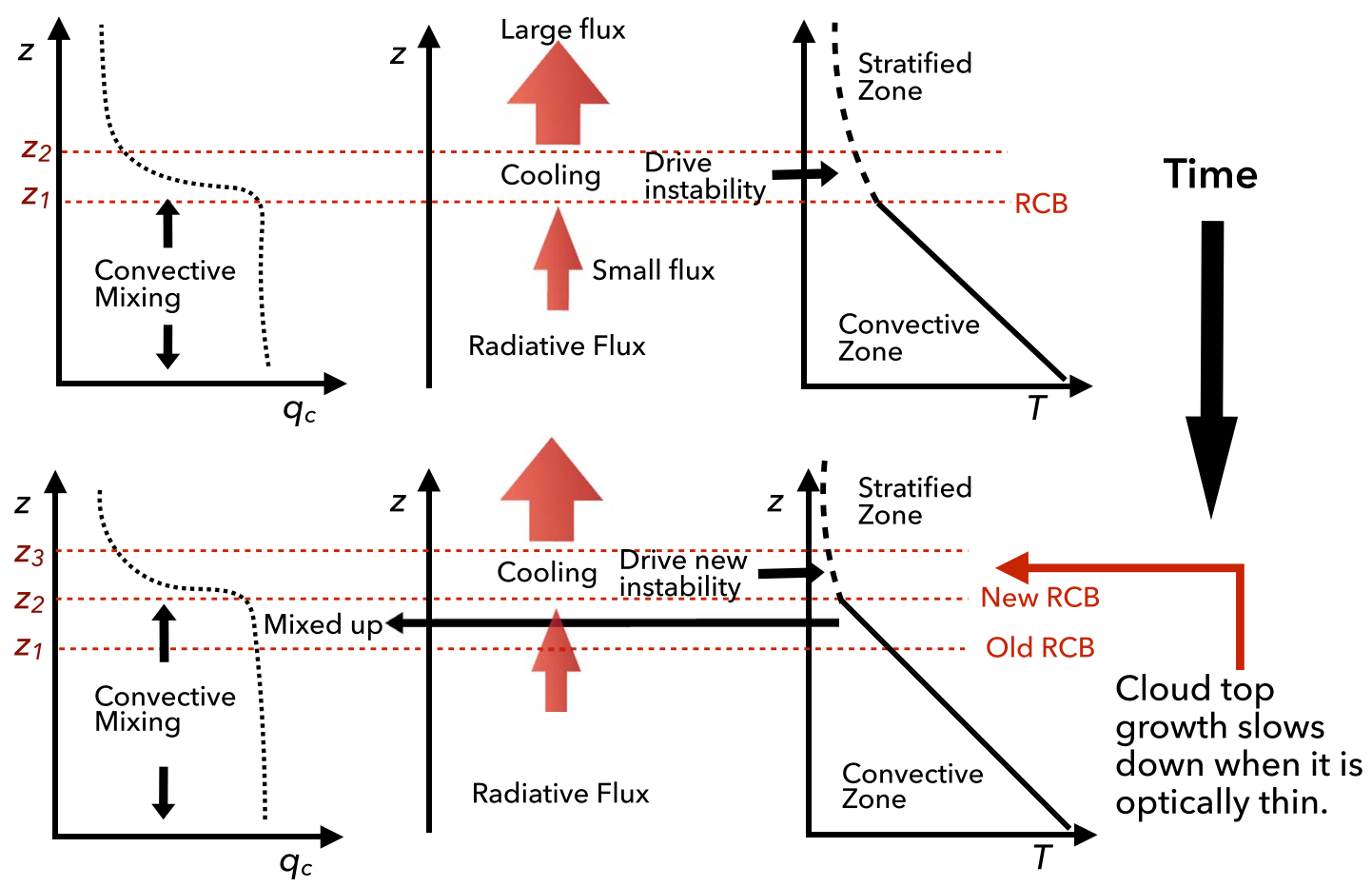

Figure 3. Illustration of the mechanism for cloud-top growth - the generation of convective instability by the vertically sharp cloud top. Suppose that initially, the cloud top, as well as the top of the secondary convective zone, lies at altitude $z_{1}$, and there exists a transition to a nearly cloud-free altitude $z_{2}$, which is slightly above $z_{1}$. Convective mixing is strong enough to almost well mix clouds up to $z_{1}$, above which mixing is too small to maintain much cloud. This results in a sharp transition of the cloud mixing ratio and thus a large opacity gradient between $z_{1}$ and $z_{2}$. This configuration drives strong cooling for the layer between $z_{1}$ and $z_{2}$. After some time, the temperature at $z_{2}$ is sufficiently cool, the layer between $z_{1}$ and $z_{2}$ becomes convectively unstable, and clouds are then mixed up to the higher altitude $z_{2}$. Therefore, a new sharp cloud top forms between $z_{2}$ and a higher altitude $z_{3}$. This procedure repeats, and the cloud top is able to extend to higher altitudes over time. The ascent of the cloud top can be slowed down or terminated either when the cloud top reaches a sufficiently high altitude where it is optically thin or when the cloud mixing ratio is sufficiently small.

substantially during the evolution and differs from the prediction of the intersection of the condensation curve described by Equation (5) and the time-mean $T-P$ profile. This is not surprising, because the actual condensation level is determined by the instantaneous vapor and temperature profiles, both of which vary during the evolution.

\subsection{Mechanism of the Variability}

The evolution of the system is mainly comprised of the formation and dissipation of clouds and the corresponding transition between the convective and stratified thermal profiles, as shown in both Figures 1 and 2. In between the cycles, the thickness of the cloud layer, as well as the altitude of the cloud top, gradually grow and then decay. Here we discuss the mechanisms that control the behavior and maintain the variability.

After the cloud layer forms, it dissipates primarily by particle settling through the cloud base. The stratification right below the cloud base plays a key role in suppressing efficient upward transport of both condensable vapor and clouds through the cloud base. Thus, there is no source to balance the loss of the total amount of tracer, including clouds and vapor in the cloudforming region, and inevitably, the cloud layer will eventually decay. The secondary convective layer that coexists with the cloud layer cannot prevent the net sinking by particle settling unless it can penetrate below the cloud base and mix up vapors. However, this is not physically feasible due to the strong heating rate at the cloud base that stratifies the regions below the cloud base, as shown in Figure 2. One could wonder whether this result could be an artifact of using diffusion to parameterize convective transport. In reality, convection has upward and downward motions. In regions of strong updrafts, cloud settling may be prevented, but in regions of downdrafts, falling out of clouds is inevitable, so there will always be net rainout through the cloud base.

After the cloud layer dissipates, the atmosphere will cool off due to the larger outgoing radiative flux. Relative to the temperature at a deeper level, which is approximately constant in time, the cooling of the upper layers lessens the stratification of the profile (i.e., makes $d \ln T / d \ln p$ larger) and eventually promotes upward convective mixing of deep vapor. In some situations, the cooling also triggers supersaturation of a substantial amount of vapor. In both cases, a new cloud deck can form, and the cycle repeats. Of course, this simplified picture does not describe the full details, such as the small subcycle seen in Figure 1, but demonstrates the qualitative governing mechanism for the cloud cycle.

Within a cloud cycle, the thickness of a cloud layer and the altitude of the cloud top grow, which is responsible for the decrease of outgoing thermal flux shown in Figure 1. This requires the secondary convective zone to continuously extend to higher and higher altitudes over time. The mechanism uplifting the top of the secondary convective zone is the continuous generation of convective instability in the layer just above the cloud top due to the large vertical gradient of the 
cloud mixing ratio near the cloud top. We depict the process using a cartoon plot in Figure 3. Suppose that initially, the cloud top, as well as the top of the secondary convective zone, lies at altitude $z_{1}$, and there exists a transition to a nearly cloudfree altitude $z_{2}$, which is slightly above $z_{1}$. Convective mixing is strong enough to almost well mix clouds up to $z_{1}$, above which mixing is too small to maintain much cloud. This results in a sharp transition of the cloud mixing ratio and thus a large opacity gradient between $z_{1}$ and $z_{2}$. This configuration drives strong cooling for the layer between $z_{1}$ and $z_{2}$ by the fact that the radiative flux is higher above $z_{2}$ than below $z_{1}$ (see the large gradients and strong cooling just above the cloud top in Figure 2$).^{5}$ After some time, the temperature at $z_{2}$ is sufficiently cool, the layer between $z_{1}$ and $z_{2}$ becomes convectively unstable, and clouds are then mixed up to the higher altitude $z_{2}$. Therefore, a new sharp cloud top forms between $z_{2}$ and a higher altitude $z_{3}$. This procedure repeats, and the cloud top is able to extend to higher altitudes over time. Another essential angle to understand this mechanism is that, as the cloud-top altitude rises, the thermal radiation to space decreases, and this cools off the entire temperature profile of the stratified, cloud-free atmosphere that is above the cloud. Because the opacity structure has a large vertical gradient near the cloud top, this cooling tends to drive a large temperature lapse rate just above the cloud top and thus causes the instability to extend slightly higher in altitude. This then cools the above-cloud temperature profile even more, causing the cloud top to extend to even higher altitude.

The ascent of the cloud top can be slowed down or terminated by two processes. First, when the cloud top reaches a sufficiently high altitude, the atmosphere becomes too optically thin to drive an instability, and therefore the growth of the cloud top ceases. ${ }^{6}$ Second, when the cloud mixing ratio is significantly reduced due to the aforementioned dissipation mechanism, the opacity gradient is reduced, and so is the top cooling. For example, row D in Figure 2 shows that the cloud top is at optical depth $\ll 1$, and there is no top cooling; row $\mathrm{E}$ shows that when the cloud mixing ratio is reduced, there is no top cooling. Clouds start dissipating when the cloud mixing ratio significantly decreases and thus becomes unable to maintain sufficient convective mixing to balance the settling. Essentially, this is a runaway process that kills off the cloudthe lessening of the cloud opacity lessens the opacity discontinuity at the cloud top, which lessens the cooling spike at the cloud top and therefore helps to inhibit the convective instability, all of which further acts to suppress upward mixing of cloud material, allowing the cloud to die by particle settling in a stagnant environment. Rainout starts from low pressure where the settling velocity is higher than at high pressure (see the settling velocity as a function of pressure in, e.g., Parmentier et al. 2013). Above the levels where clouds dissipate, air is warmed up by the larger upwelling radiative flux, thus stratifying those levels.

\footnotetext{
In the presence of a large opacity gradient that increases with depth, the atmospheric profile is usually convectively unstable if it is in radiative equilibrium (see a discussion in, e.g., Rauscher \& Menou 2012). A larger opacity gradient leads to a more unstable equilibrium profile. In the nonequilibrium atmosphere with a strong opacity gradient, like the layer near the cloud top in our case, radiation tends to drive the atmosphere toward a convectively unstable equilibrium by strong top cooling.

6 In radiative equilibrium, the atmosphere is stratified in the optically thin limit even in the presence of a strong opacity gradient. In this limit, the cooling that drives the atmosphere toward equilibrium cannot generate convective instability.
}
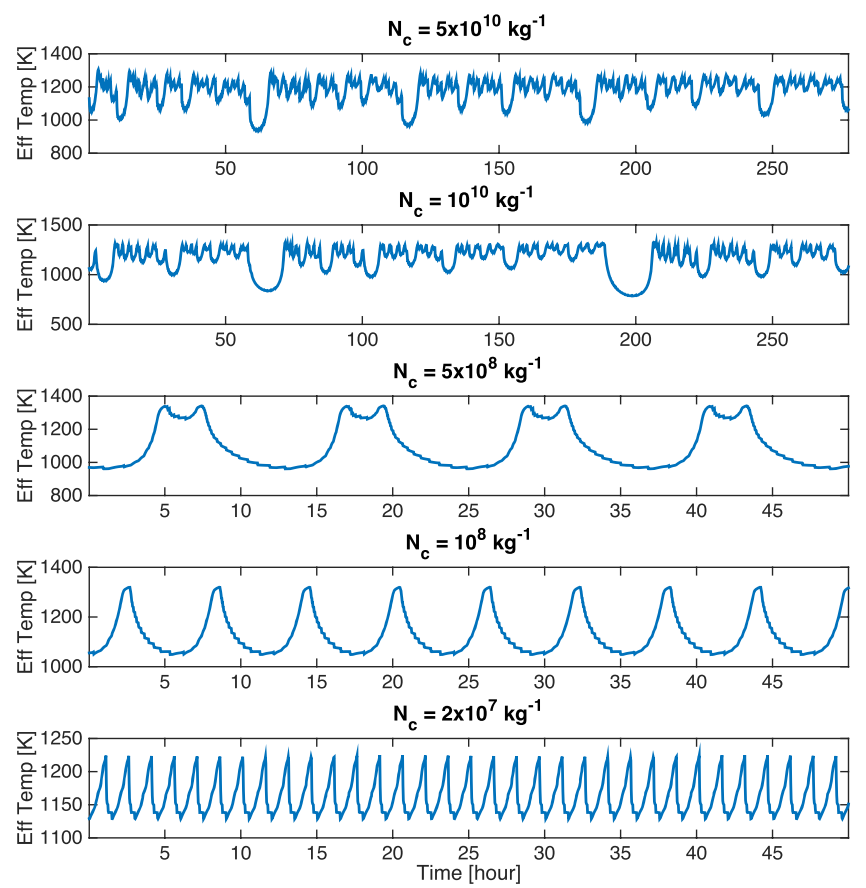

Figure 4. Outgoing flux plotted as effective temperature as a function of time for cases with cloud number density $\mathcal{N}_{c}=5 \times 10^{10}, 10^{10}, 5 \times 10^{8}$ (the nominal model), $10^{8}$, and $2 \times 10^{7} \mathrm{~kg}^{-1}$. Other model parameters are the same as in the nominal case in Section 3.1.

The spontaneous and continuous variability of the system is therefore maintained by the persistent imbalances. Because of the imbalanced net cloud settling, the cloud layer will continuously dissipate until it is replenished by a new cloud deck. Also, because the cloud layer and the secondary convective zone are tightly coupled, the evolution of clouds forces the evolution of the thermal structure. The imbalance of clouds is likely inevitable because the secondary convective zone cannot extend below the cloud base. Thus, the system shown in Figures 1 and 2 is intrinsically variable.

\subsection{Sensitivity Studies}

We study the sensitivity of the variability to various model assumptions and show that the detailed evolution is sensitive to model parameters, some of which are in reality rather unconstrained. However, the existence of variability is robust over a wide range of model assumptions. In the following sections, only the mentioned parameters are changed, and other parameters are the same as in the nominal case. We discuss some important cases in detail but leave out the details of most cases to avoid redundancy.

\subsubsection{Cloud Number Density}

The particle number per mass $\mathcal{N}_{c}$ controls the particle sizes and cloud opacities, determining the cloud settling flux and heating/cooling rate. We perform a series of models with $\mathcal{N}_{c}=2 \times 10^{7}$ to $5 \times 10^{10} \mathrm{~kg}^{-1}$, which is a significant variation around the nominal value $5 \times 10^{8} \mathrm{~kg}^{-1}$. To give a sense, assuming a cloud mixing ratio $10^{-3} \mathrm{~kg} \mathrm{~kg}^{-1}$, the peak of the particle size distribution is at about 1.3 and $0.1 \mu \mathrm{m}$ for $\mathcal{N}_{c}=2 \times 10^{7}$ and $5 \times 10^{10} \mathrm{~kg}^{-1}$, respectively. Figure 4 shows the outgoing thermal flux in terms of effective temperature as a function of time. Strikingly, the high- $\mathcal{N}_{c}$ 

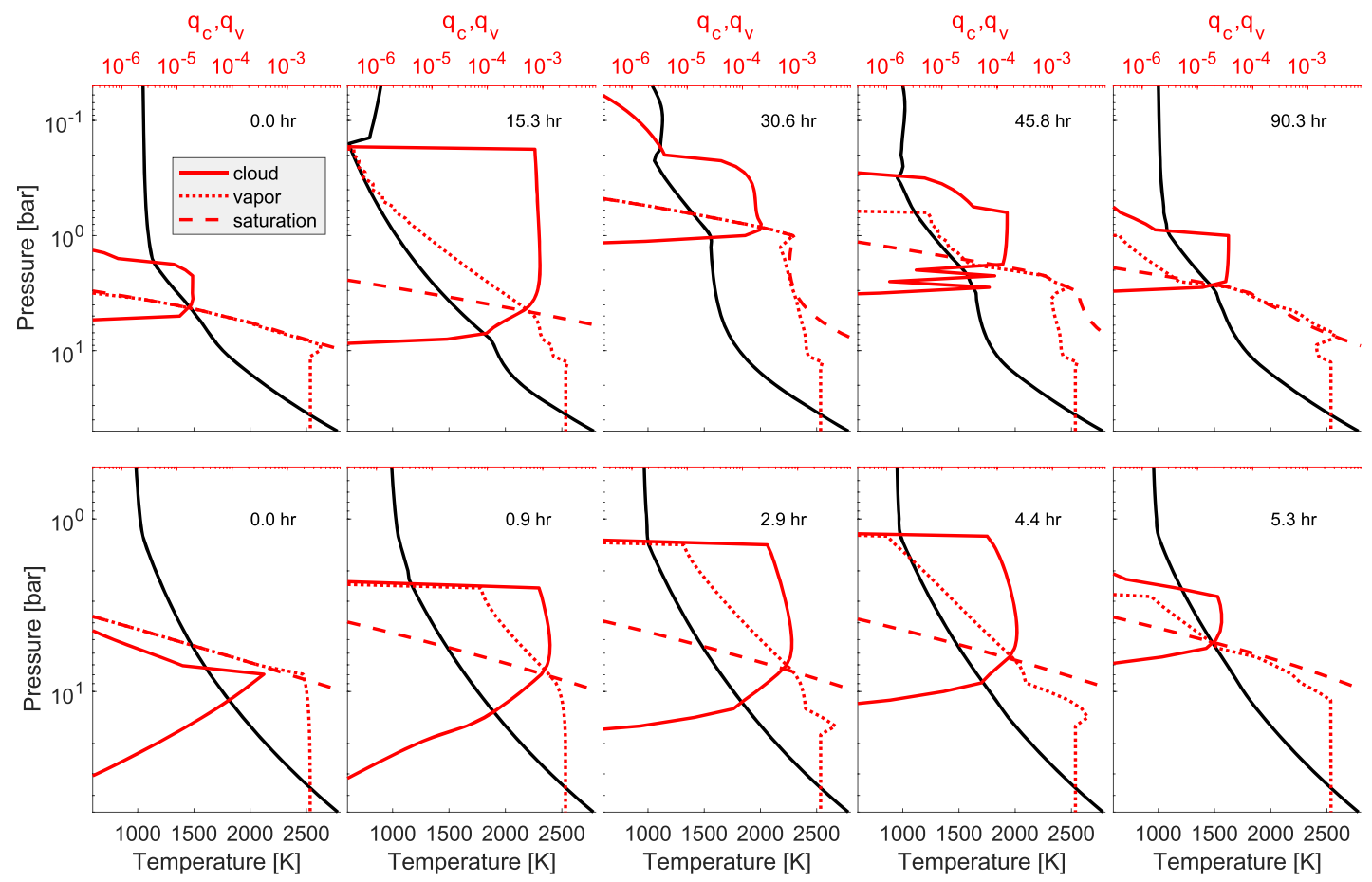

Figure 5. Snapshots of temperature, vapor, cloud mixing ratio, and saturation vapor mixing ratio for models with cloud number per mass $\mathcal{N}_{c}=5 \times 10^{10}$ in the first row and $10^{8} \mathrm{~kg}^{-1}$ in the second row (a factor of 100 times greater and 5 times less than the nominal value, respectively). The black curve represents temperature (bottom abscissa), and the red curves represent cloud, vapor, and saturation vapor mixing ratios (top abscissa). Time increases from left to right starting from zero, as shown in the panels.

cases $\left(\mathcal{N}_{c}=5 \times 10^{10}\right.$ and $\left.10^{10} \mathrm{~kg}^{-1}\right)$ exhibit irregular variabilities as opposed to models with number densities smaller than $5 \times 10^{8} \mathrm{~kg}^{-1}$ that exhibit quasi-periodic variabilities. A clear trend for the low-number-density models is the decreasing oscillation period with decreasing $\mathcal{N}_{c}$. The decrease of $\mathcal{N}_{c}$ causes the peak of the size spectrum to shift to larger particles and thus a larger settling flux. If the change of size spectrum is gradual (i.e., the corresponding cloud extinction coefficient distribution does not change drastically), the decrease of $\mathcal{N}_{c}$ reduces the cloud opacity. Qualitatively, both effects reduce the time needed to complete a cloud cycle.

The transition from regular to irregular variability with increasing cloud number density is due to the onset of chaos. The evolution of the system is sensitive to initial conditions when $\mathcal{N}_{c}$ is high. We perform experiments of slightly different initial conditions for the model with $\mathcal{N}_{c}=5 \times 10^{10} \mathrm{~kg}^{-1}$ by perturbing just $1 \%$ of the cloud mixing ratio in one grid point. The initial condition of this experiment is taken from an instantaneous output of an $\mathcal{N}_{c}=5 \times 10^{10} \mathrm{~kg}^{-1}$ model after reaching a statistical equilibrium. The evolution of the two cases shows drastically different trajectories after some time, as shown in the upper right panel of Figure 13 in Appendix B. On the contrary, experiments with vastly different initial cloud structures for the model with $\mathcal{N}_{c}=10^{8} \mathrm{~kg}^{-1}$ show a quick merging of the evolution to the regular periodic state after some differences at the very beginning. The sensitive dependence of high- $\mathcal{N}_{c}$ models is the root cause of irregularity shown in the evolution of the systems (e.g., Motter \& Campbell 2013).

Chaos can emerge from a forced-dissipated nonlinear system (Lorenz 1963). In our system, the qualitative condition under which chaos may occur is likely that the cloud radiative heating should be able to strongly alter the temperature structure, such that the saturation vapor mixing ratio profile can evolve significantly. On the other hand, in conditions showing quasi-regular variabilities, the variation of temperature near the cloud base is quite small, so the saturation vapor mixing ratio profile stays roughly unchanged. In the latter condition, the cloud cycle is controlled by the processes of settling and stratification (which only needs a slight change of thermal structure), which are well described by the ideal picture in the mechanism (Section 3.2). In essence, a new cloud cycle starts only when the stratification disappears. In this sense, the system is simple and "linear." The system can become sufficiently "nonlinear" when the saturation vapor mixing ratio can significantly evolve. Formation of clouds can then be additionally triggered by cooling of the thermal structure that supersaturates the local vapor. The varying saturation vapor mixing ratio serves as an extra degree of freedom to the system that is nonlinearly coupled to other variables, leading to strong "nonlinearity," a preferred condition for the emergence of chaos. To illustrate the above two conditions, Figure 5 presents several snapshots of the temperature, cloud, vapor, and saturation vapor profiles for the model with $\mathcal{N}_{c}=5 \times$ $10^{10} \mathrm{~kg}^{-1}$ in the upper row and $\mathcal{N}_{c}=10^{8} \mathrm{~kg}^{-1}$ in the lower row. Because the model with $\mathcal{N}_{c}=5 \times 10^{10} \mathrm{~kg}^{-1}$ is irregular, the snapshots are randomly chosen, but the snapshots for the model with $\mathcal{N}_{c}=10^{8} \mathrm{~kg}^{-1}$ sample a full single cloud cycle. We can obviously see that in the large- $\mathcal{N}_{c}$ model, all profiles evolve substantially. Below the cloud base, the vapor profile is sometimes quite close to the saturation vapor profile, so that any slight cooling can trigger cloud formation (for example, the spiky cloud structure seen below the main cloud deck in the fourth upper panel). On the contrary, in the low- $\mathcal{N}_{c}$ model, the saturation vapor pressure curve barely varies. In fact, all models explored in this study have either quasi-regular or irregular variability, and their evolutions all fall into either of the two categories shown in Figure 5. 

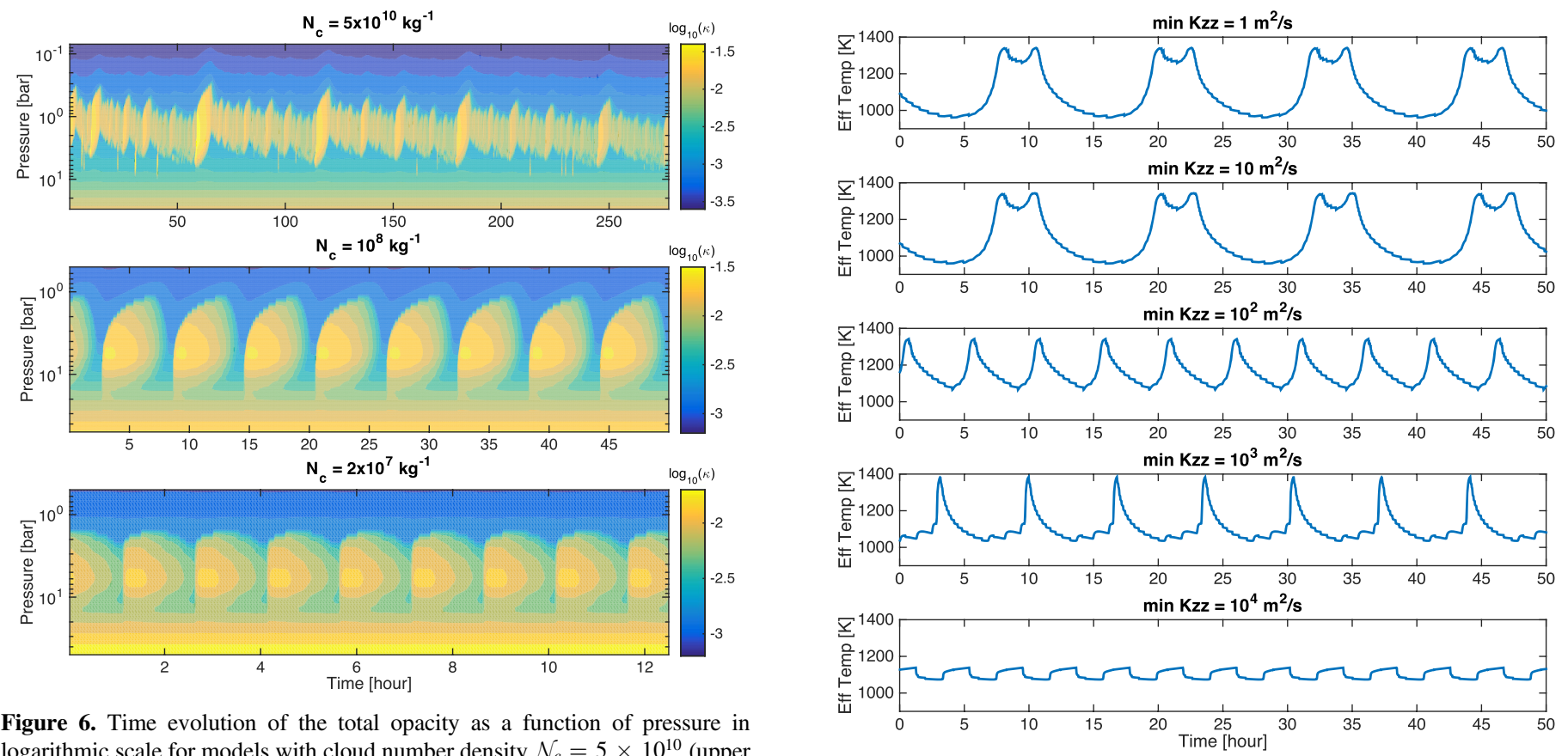

Figure 6. Time evolution of the total opacity as a function of pressure in logarithmic scale for models with cloud number density $\mathcal{N}_{c}=5 \times 10^{10}$ (upper panel), $10^{8} \mathrm{~kg}^{-1}$ (middle panel), and $2 \times 10^{7} \mathrm{~kg}^{-1}$ (lower panel).

Interestingly, the high- $\mathcal{N}_{c}$ models show cloud coverage almost all the time during the evolution, as opposed to the relatively low$\mathcal{N}_{c}$ models in which cloud opacities sometimes contribute negligibly to the total opacity. We show the total opacity structure as a function of time in Figure 6 for the models with $\mathcal{N}_{c}=5 \times 10^{10}$ in the upper panel, $10^{8} \mathrm{~kg}^{-1}$ in the middle panel, and $2 \times 10^{7} \mathrm{~kg}^{-1}$ in the lower panel. The background gaseous opacity generally decreases with decreasing pressure, so it is easy to recognize the cloud opacity as those local maximums evolving near the cloud-forming region. The high- $\mathcal{N}_{c}$ model shows no cloud opacity gap in the evolution, while there is an obvious opacity gap in the medium- $\mathcal{N}_{c}$ model but then almost no gap again in the low- $\mathcal{N}_{c}$ model. Qualitatively, cloud cycles in the high- $\mathcal{N}_{c}$ models do not rely on a complete dissipation of clouds but instead are mainly driven by heating/cooling of the thermal profile, so that we rarely see a cloud-free instant in these models. In the medium- $\mathcal{N}_{c}$ model, cloud opacity has a much smaller impact on the thermal structure, and thus a nearly full dissipation of the cloud deck is allowed. In the case of very low $\mathcal{N}_{c}$, the duration of stratification is too short because of less cloud radiative warming, and the new cloud cycle is quickly triggered before the old cloud deck fully dissipates. We will discuss the implications in the discussion section.

\subsubsection{The Background Tracer Diffusion}

Vertical transport of tracers could occur in the stratified atmospheres via, for instance, shear instability, wave breaking, and large-scale winds. It is difficult to quantify these effects without a detailed understanding of the atmospheres. Instead, we explore the effect of mixing in the stratified layer by simply imposing an additional constant background diffusion $K_{\mathrm{zz}, \min }$ for tracers, such that the total diffusion coefficient is $\max \left[K_{\mathrm{zz}}, K_{\mathrm{zz}, \mathrm{min}}\right]$, with the former determined by Equation (10). We explore values from $K_{\mathrm{zz}, \min }=1$ to $10^{4} \mathrm{~m}^{2} \mathrm{~s}^{-1}$. Figure 7 shows their effective temperature as a function of time. All models show variability in the thermal flux. Cases with relatively small $K_{\mathrm{zz}, \min }\left(1\right.$ and $\left.10 \mathrm{~m}^{2} \mathrm{~s}^{-1}\right)$

Figure 7. Outgoing thermal flux in terms of effective temperature as a function of time for models with a constant minimal tracer diffusion coefficient $K_{\mathrm{zz}, \min }=1,10,10^{2}, 10^{3}$, and $10^{4} \mathrm{~m}^{2} \mathrm{~s}^{-1}$. Other parameters are the same as the nominal model.

have both oscillation periods and flux variation amplitudes quantitatively similar to the nominal case. Cases with medium values $\left(10^{2}\right.$ and $\left.10^{3} \mathrm{~m}^{2} \mathrm{~s}^{-1}\right)$ display quantitatively different evolutions but still retain large variations and somewhat close oscillation periods as the nominal model. The case with $K_{\mathrm{zz}, \min }=10^{4} \mathrm{~m}^{2} \mathrm{~s}^{-1}$ exhibits a higher oscillation frequency and a much smaller variation amplitude.

We conclude that a medium background diffusion is unlikely to suppress the variability under the parameter regime of the nominal model. However, the details can be affected. Roughly speaking, nontrivial background diffusion can efficiently mix deep vapor upward and constantly promote cloud formation near the cloud base. This is different from the nominal model, where the supply of deep vapor is terminated once the atmosphere below the cloud base is stratified. Compared to the nominal model, this brings the temperature profile below the cloud base closer to the condensation curve and uplifts the cloud base to a higher altitude because of cloud radiative heating. The particle settling strength is higher at lower pressure and thus partly contributes to the subtly different evolutionary details seen in Figure 7. For sufficiently large background diffusion that can completely balance the particle settling, the variability could be suppressed, and the system can approach a steady state. Values of the diffusion coefficient $K_{\mathrm{zz}}$ derived from fitting to spectra of field BDs using nonequilibrium chemical models are moderate $\left(\sim 10^{2} \mathrm{~m}^{2} \mathrm{~s}^{-1}\right.$ or $\sim 10^{6} \mathrm{~cm}^{2} \mathrm{~s}^{-1}$; e.g., Stephens et al. 2009) for many $\mathrm{L}$ and $\mathrm{T}$ dwarfs, which is unlikely to suppress variability in the parameter regime similar to our nominal model.

\subsubsection{Conversion Timescale}

One interesting feature in the nominal model is that the supersaturation can be orders of magnitude larger than $100 \%$ (see Figure 2). In Earth's atmosphere, the supersaturation of 

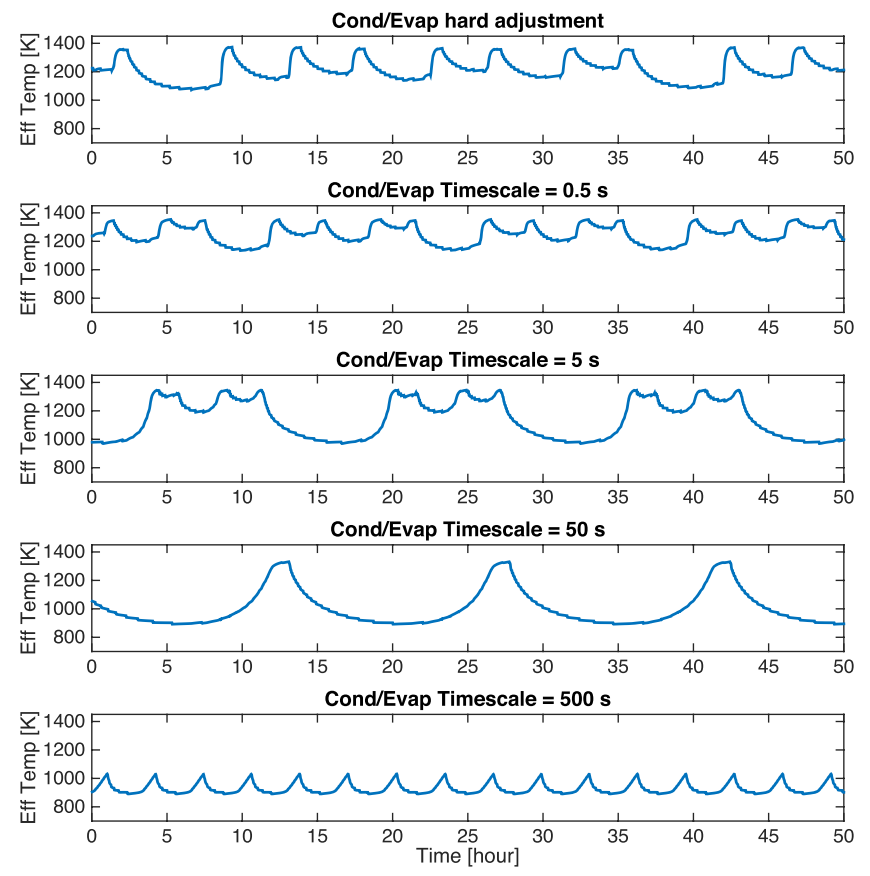

Figure 8. Outgoing thermal flux in terms of effective temperature as a function of time for cases with different conversion timescales $\tau_{c}=$ infinitely small, $0.5,5,50$, and $500 \mathrm{~s}$. In the case of $\tau_{c}=$ infinitely small, we did a hard adjustment for conversion; i.e., the vapor/cloud mixing ratio is instantaneously adjusted such that no supersaturation/clouds in unsaturated air is allowed.

water seldom exceeds $1 \%$ (e.g., Houze 2014) because proper cloud condensation nuclei (CCNs) enhance the nucleation process. Even without proper CCNs, the homogeneous nucleation rate increases nonlinearly with increasing supersaturation (e.g., chapter 3, Fletcher 2011) and tends to prevent the atmosphere from arriving at such high supersaturation. ${ }^{7}$ However, this may not be the case in BD atmospheres (Helling \& Fomins 2013), and detailed cloud microphysics models suggest that supersaturation up to several orders of magnitude larger than unity is possible in these atmospheres (see Figure 9 in Helling \& Casewell 2014). In any case, we examine whether the existence of variability relies on high supersaturation and how variability behaves with varying supersaturation. This is done simply by adjusting the conversion timescale $\tau_{c}$, which mainly controls the degree of supersaturation as shown in Equation (11). Figure 8 shows outgoing flux from models with $\tau_{c}=$ infinitely small, $0.5,5,50$, and $500 \mathrm{~s}$. In the case of infinitely small $\tau_{c}$, we perform a "hard" adjustment for conversion between vapor and cloud. This means that whenever there is supersaturation for vapor or cloud in a subsaturated environment, we instantaneously adjust the vapor and cloud to an equilibrium state without using a relaxation scheme. Variability emerges from all models, suggesting that the degree of supersaturation within a reasonable range does not affect the existence of variability. Models with $\tau_{c} \leqslant 50 \mathrm{~s}$, although differing in quantitative details, exhibit qualitatively similar temporal evolution. This is because the strong convective mixing is able to mix the bulk tracers $\left(q_{v}+q_{c}\right)$ against cloud settling, and the detailed conversion between vapor and clouds does not affect the overall mechanisms driving the variability. The model with $\tau_{c}=500 \mathrm{~s}$ shows

\footnotetext{
7 For instance, $\sim 300 \%$ supersaturation is sufficient to trigger efficient homogeneous nucleation of water vapor in Earth's troposphere (e.g., Houze 2014).
}

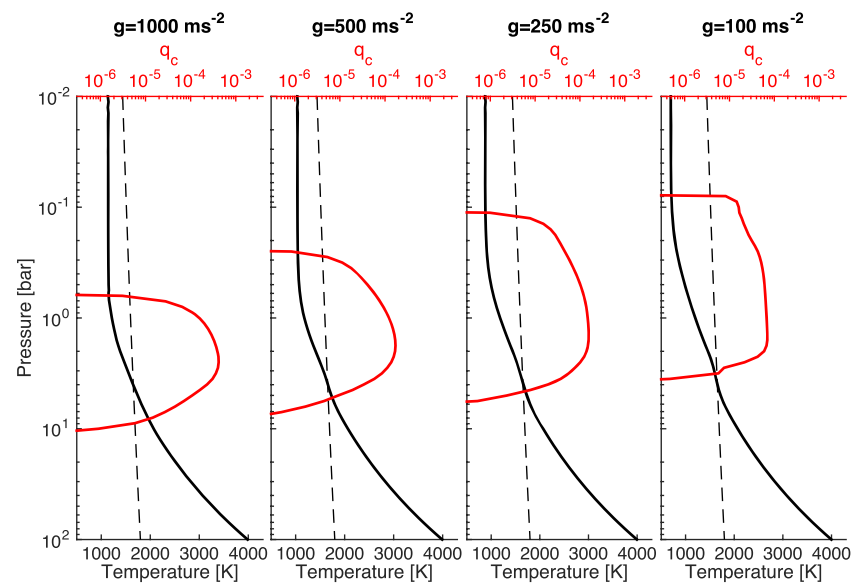

Figure 9. Time-averaged temperature and cloud structure for models with different surface gravity $g=1000,500,250$, and $100 \mathrm{~m} \mathrm{~s}^{-2}$, and all models have a temperature of $4000 \mathrm{~K}$ at the model bottom boundary of 100 bars.
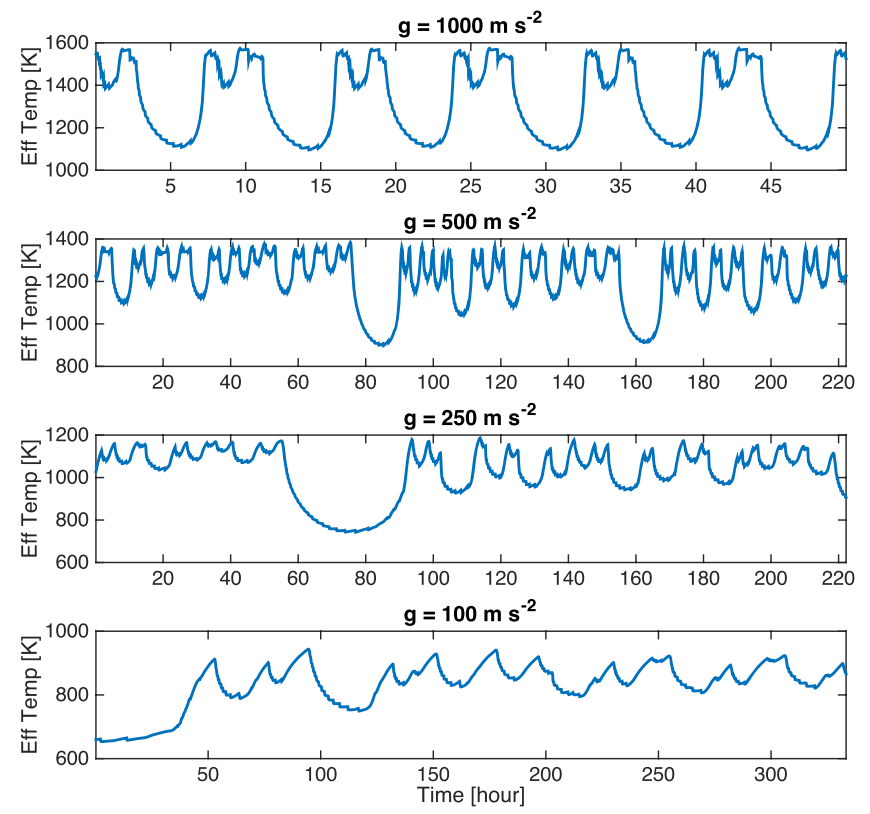

Figure 10. Outgoing thermal flux in terms of effective temperature as a function of time for models with different surface gravity $g=1000,500,250$, and $100 \mathrm{~m} \mathrm{~s}^{-2}$. All models have a temperature of $4000 \mathrm{~K}$ at the model bottom boundary of 100 bars.

smaller average flux and variation amplitude. In this case, abundant vapor can be maintained throughout the cloudforming region almost all the time. Thus, the main cloud deck is thick and has little variability due to supply from the invariant vapor profile. The main variability contributing to the outgoing flux is by the slightly varying cloud-top altitudes.

\subsubsection{Surface Gravity}

Variability has also been detected in relatively low-gravity objects, including directly imaged EGPs and free-floating planetary-mass objects (Biller et al. 2015; Zhou et al. 2016; Gagné et al. 2017), although it is yet unclear for these objects whether the surface features evolve on a short timescale in a similar fashion as many field BDs (Metchev et al. 2015; Apai et al. 2017). Changes in the surface gravity affect the cloud 

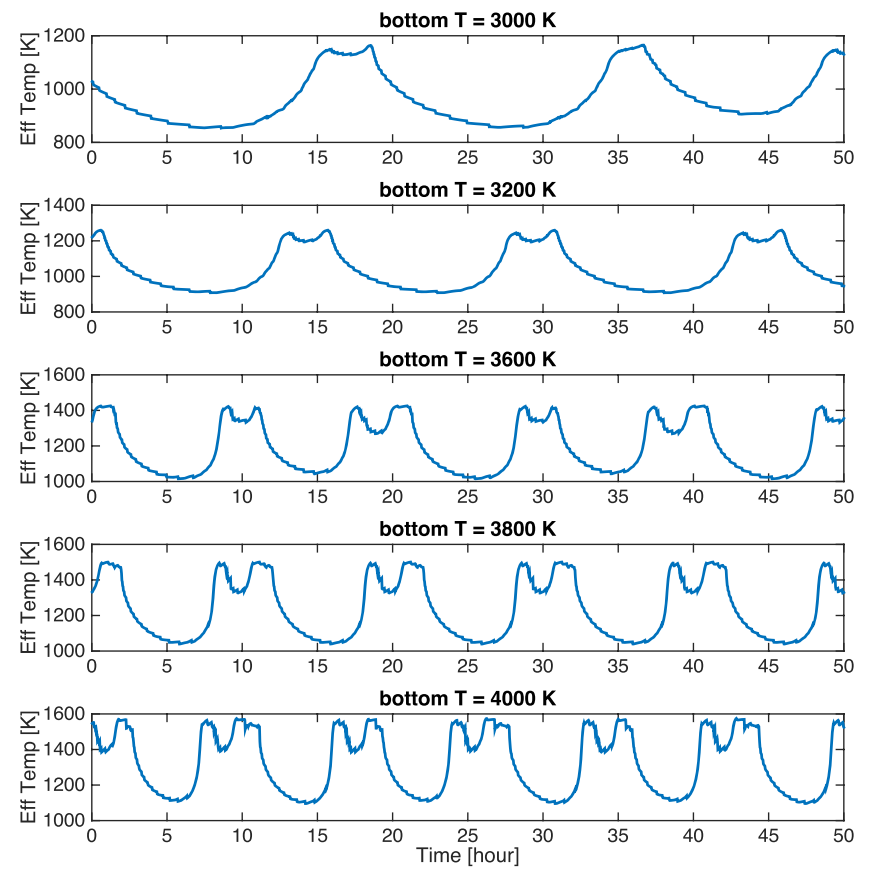

Figure 11. Outgoing thermal flux in terms of effective temperature as a function of time for models with different temperatures $T_{b}=3000,3200$, 3600,3800 , and $4000 \mathrm{~K}$ at the bottom boundary 100 bars.

settling velocity, convective mixing, and $T-P$ structure by changing the optical depth. But we expect that the changes of gravity do not affect the fundamental driving mechanisms of variability. We carry out simulations with gravity $g=1000$, 500,250 , and $100 \mathrm{~m} \mathrm{~s}^{-2}$, and all models have a temperature of $4000 \mathrm{~K}$ at the model bottom boundary of 100 bars. Figure 9 shows the time-averaged $T-P$ and cloud mixing ratio profiles, and Figure 10 shows the outgoing flux for the models. If there there were no radiative cloud feedback, the pressure of the cloud base would be the same for all models because the intersections of the $T-P$ profile and the condensation curve are in the convective layer. With decreasing gravity, the atmospheres undergo greater back-warming below the cloud base due to the lower settling velocity in lower-gravity conditions. This results in the higher-altitude time-averaged cloud base in the lower-gravity cases. As discussed in Section 3.2, the altitude of the cloud top is roughly where the optical depth transitions to $\ll 1$. If the background gaseous opacity is not very sensitive to temperature, the pressure of the cloud top roughly scales as gravity, which can loosely explain the trend of time-averaged cloud-top pressures $(0.6,0.27,0.13$, and 0.08 bar for $g=1000,500,250$, and $100 \mathrm{~m} \mathrm{~s}^{-2}$, respectively) shown in Figure 9. The outgoing flux of the $g=1000 \mathrm{~m} \mathrm{~s}^{-2}$ model is quasi-periodic, but the fluxes are irregular for lowergravity models due to the onset of chaos. As discussed above, the onset of chaos in low-gravity models is due to the sufficient temperature change by cloud radiative heating, as demonstrated in Figure 9.

\subsubsection{Atmospheric Temperature}

Variability is common among field $\mathrm{L}$ and $\mathrm{T}$ dwarfs across a wide range of atmospheric temperatures. Different temperatures affect the pressure level of first condensation, and thus the cloud settling velocity and radiative heating/cooling rate. Here we show experiments in which we change the temperature
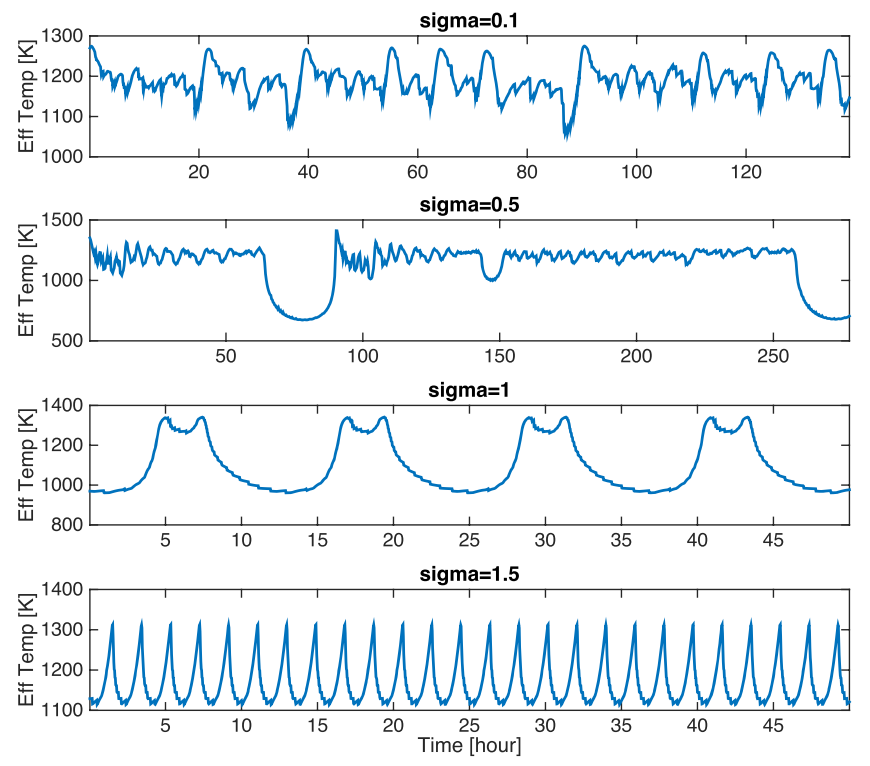

Figure 12. Outgoing thermal flux in terms of effective temperature as a function of time for models with $\sigma=0.1,0.5,1$, and 1.5. Here $\sigma$ is a parameter that controls the width of the cloud particle size distribution.

$T_{b}=3000,3200,3600,3800$, and $4000 \mathrm{~K}$ (note that $3400 \mathrm{~K}$ is the value adopted for the nominal model) at the bottom boundary 100 bars in Figure 11. All of the models show quasiperiodic variability and an obvious trend: the oscillation frequency is higher for hotter models. Qualitatively, two factors are responsible. First, in hotter models, the cloud layer dissipates faster after it forms due to the lower-pressure cloud base and thus the higher settling velocities. Second, in hotter models, the cloud-forming region cools down faster after the cloud dissipates due to the shorter thermal relaxation timescale, helping to restore the next cloud cycle in a shorter timescale.

\subsubsection{Shape of Size Distribution Function}

We perform a sequence of models varying parameter $\sigma$ controlling the width of the lognormal size distribution of cloud particles to examine the sensitivity to the shape of the sizedistribution function. Figure 12 shows outgoing fluxes of models with $\sigma=0.1,0.5,1$, and 1.5 . Models with relatively narrow size distribution ( $\sigma=0.1$ and 0.5 ) show irregular variability. In these cases, the settling flux is relatively small compared to large- $\sigma$ models because of the smaller fraction of large particles. The model with $\sigma=1.5$ is regular and oscillates with a much shorter period than the model with $\sigma=1$ due to a much larger settling flux. As discussed in Section 2, models with exponential cloud size distribution also exhibit variabilities qualitatively similar to the lognormal distribution. We conclude that details of the cloud size distribution strongly affect the quantitative behavior of the variability, but the mechanisms remain the same.

\section{Discussion}

\subsection{Under What Conditions Does the 1D Cloud-driven Variability Not Occur?}

It is impractical to explore the variability through the whole parameter space, and we qualitatively discuss conditions under which the 1D system governed by Equations (1)-(3) may have little variability. We list several, but probably not all, such conditions. (1) Vapor first condenses in the stratified layer, no 
background mixing, and relatively large cloud particles. Under these conditions, the cloud layer will quickly dissipate via particle settling but cannot be replenished by vapor transport because the atmosphere is stratified and there is no background mixing. (2) Strong background mixing and small cloud particles. Very strong background mixing is able to completely balance particle settling, suppressing the cloud dissipation cycle. (3) Condensable vapor mixing ratio is too small. In this case, clouds may be too optically thin to effectively alter the thermal structure and maintain the cloud cycle. Considering realistic atmospheric conditions from mid-L to mid-T dwarfs, including directly imaged EGPs, the conditions leading to no variability seem stringent. Perhaps late-T dwarfs represent conditions under which cloud-driven variability does not easily occur, at least in our simple 1D context, because major condensates such as silicates and iron condense at deep layers where the background gaseous opacity is too large for cloud opacities to matter much. Indeed, cloud-free models can interpret spectra of late-T dwarfs reasonably well (e.g., Line et al. 2017). This suggests that our mechanism should apply to the majority of BDs and directly imaged EGPs for which reasonably thick clouds are expected to exist in the observable atmospheres.

\subsection{Implications for Observations}

The radiative cloud-driven variability provides a theoretical foundation to understand the rapidly evolving light curves found for many L and T dwarfs (e.g., Artigau et al. 2009; Metchev et al. 2015; Apai et al. 2017). These observations are difficult to explain by rotational modulation caused by temporally steady or slowly evolving surface inhomogeneity (Karalidi et al. 2016), for example, phenomena analogous to the Great Red Spot or $5 \mu \mathrm{m}$ hot spots in Jupiter's atmosphere. Light curves of many BDs can only be explained when the surface features evolve over timescales comparable to the rotation period. Our results show that the timescale of the cloud-driven variability can be as short as a few hours, depending on the model parameters. If the surface features are driven by such variability, the rotation-modulated light curves will be irregular over rotational timescales. Moreover, even if the $\mathrm{BD}$ is observed from a nearly pole-on geometry-which minimizes the effect of rotational modulation in the light curves - short-term evolution of light curves could still occur due to the evolving statistics of surface patches driven by variability caused with the mechanism explored here. Nearly pole-on variable BDs could be valuable targets to characterize the intrinsic variability driven by clouds. Our models show that thick clouds usually correlate to a convective and cooler profile, and thin clouds correlate to a stratified and warmer profile, which is a result of coevolution of clouds and thermal structure. For some variable BDs characterized at multiple wavelengths, a combination of two types of surfaces consisting of a warm, thin-cloud patch and a cool, thick-cloud patch is necessary to fit the observations (Apai et al. 2013; Buenzli et al. 2015). Our model provides a mechanism for such a combination of surface features. In our model, temperature variation exhibits a pressure-dependent shift, and the maximum difference is between the cloud top and base (see the upper panel in Figure 1). This may shed light on the observed offsets of wavelength-dependent light curves for some variable BDs (Buenzli et al. 2012; Yang et al. 2016). Our simple gray model here only reveals the mechanisms, and more sophisticated nongray models with proper chemistry are needed to quantify the spectroscopically resolved variability.
The characteristic evolution of clouds in models with relatively large particle sizes (see Figures 1 and 6) provides a mechanism for cloud breaking, in which clouds sometimes almost fully dissipate and contribute negligibly to the total opacity. Statistically, the surface cloud patches governed by such cloud cycles must have some portion occupying the cloud-dissipated state. As a result, the globally integrated outgoing flux of the atmosphere is only partially affected by cloud opacity. On the other hand, in models with relatively small particle sizes, full cloud dissipation does not easily occur, and the cloud opacity always contributes significantly to the total opacity. As a result, the integrated outgoing flux of atmospheres with small particles is likely significantly affected by cloud opacity. This has an important application to the longstanding issue associated with the L-to-T dwarf transition that shows a sudden change of near-IR colors over a narrow effective temperature range, the $J$-band brightening, and resurgence of the gaseous FeH bands (e.g., Burgasser et al. 2002; Kirkpatrick 2005). Cloud breaking remains the most promising mechanism to explain the L/T transition (Ackerman \& Marley 2001; Burgasser et al. 2002; Marley et al. 2010). However, exactly why and how clouds break remains unclear. The working mechanisms and detailed evolution of cloud cycles shown in this work support this idea in a natural way: by interpreting observations using atmospheric and radiative transfer models, it becomes clear that early-to-mid-L dwarfs are likely dominated by submicron particles (e.g., Allard et al. 2001; Hiranaka et al. 2016; Burningham et al. 2017), and T dwarfs are reasonably well represented by large cloud particle models (e.g., Saumon \& Marley 2008). Given that the transition from $\mathrm{L}$ to $\mathrm{T}$ spectral type is accompanied by a change from small to large particles, cloud breaking starting at the $\mathrm{L} / \mathrm{T}$ transition is then a natural outcome from this sequence, as cloud breaking in time variability operates only when particles become relatively large. The condition for cloud breaking depends primarily on particles size but is rather insensitive to atmospheric temperature. Thus, in principle, the onset of cloud breaking can occur over a narrow effective temperature range. In addition, our models with larger particles usually have cooler atmospheres above the condensation level than those with smaller particles, promoting the conversion from $\mathrm{CO}$ to $\mathrm{CH}_{4}$.

\subsection{Comparison with Other Cloud Models}

The most distinctive features of our 1D model compared to other prevalent parameterized cloud models for substellar atmospheres (Ackerman \& Marley 2001; Allard et al. 2001; Tsuji 2002; Cooper et al. 2003; Burrows et al. 2006; Charnay et al. 2018) are the relaxation of exact balance on both thermal structure and clouds at any given time and self-consistent coupling between thermal structure, mixing, and clouds. These treatments bring new insights to understanding clouds in substellar atmospheres. Our results demonstrate that properties of a single cloud layer can have substantial variation over a short timescale, with major changes in the cloud mass loading, altitudes of the cloud base and top (thus the layer thickness), and the particle size distribution. The two-dimensional hydrodynamic cloud formation model by Freytag et al. (2010) is a good point of reference; unfortunately, Freytag et al. (2010) explored only regimes of small particles and did not discuss details of the variability, preventing comparisons. As discussed in Section 3.1, a single set of statistically averaged temperature and cloud profiles cannot represent the equilibrium state of atmospheres with vigorous cloud formation. Retrieval methods (e.g., Line et al. 2017) have shown that 
a single set of radiative-convective equilibrium profiles can well represent the spectra of many late-T dwarfs whose atmospheres are relatively cloud-free. It would be interesting to extend this type of study to samples of late- $\mathrm{L}$ and early- $\mathrm{T}$ dwarfs.

A first-principle cloud microphysics approach by Helling et al. in a series of papers (here we refer to Helling's model, e.g., Helling et al. 2001, 2008a; Lee et al. 2016; Lines et al. 2018; see Helling \& Fomins 2013; Helling \& Casewell 2014 for reviews) considers that CCNs are available only through homogeneous nucleation of certain species $\left(\mathrm{TiO}_{2}\right.$ in their models) and require extremely high supersaturation; i.e., the nucleation occurs at much higher altitudes above the condensation level predicted by the equilibrium chemistry. Clouds then form through heterogeneous condensation of various condensable gases upon $\mathrm{TiO}_{2}$ seed particles that fall down from the upper atmosphere. The resulting cloud structure consists of "dirty grains" with a continuous distribution from upper down to deeper atmospheres until the temperatures become sufficient for the particles to evaporate. A crucial assumption in their model is the continuous, vigorous convective transport of condensable vapor upward into nucleation regions regardless of the atmospheric conditionswhether it is stratified or convective (Woitke \& Helling 2004). As shown in this study, an important consequence of cloud formation is the stratification below the cloud base and the suppression of upward convective mixing of vapors (though some mixing may still be possible through eddies in the stratified zone). This should have a significant impact on the nucleation rate in the upper atmosphere and thus the consequent cloud formation following the seed particles. One can imagine that the nucleation in the upper atmosphere can then evolve following the evolving stratification below the cloud base, which should add additional complexity to the variability, beyond the mechanisms explored in this study. Along a similar line, Gao et al. (2018) and Powell et al. (2018) presented bin-resolved cloud microphysics models showing that convective mixing is an important parameter controlling the cloud properties. Likewise, our results suggest that the variability should have a significant impact on the detailed cloud microphysics in BD atmospheres. Interestingly, using microphysical models, Gao et al. (2014) showed that cloud properties in Venus's atmosphere could exhibit long-term variability (over timescales of months), and the variability is caused by mechanisms in the microphysical level. If such microphysical mechanisms occur in BDs and directly imaged EGPs, the coupling to the mechanism presented in this study would suggest a much more complicated situation in these atmospheres.

In reality, clouds in BDs may be composed of several cloud layers with different compositions as argued by equilibrium chemical models (e.g., Visscher et al. 2006, 2010). We expect that this would not suppress the variability, which we have explored in this work using only the condensation properties of the enstatite $\left(\mathrm{MgSiO}_{3}\right)$ cloud. From mid-L to mid-T dwarfs, the most abundant cloud species that dominate the heating/cooling are silicate and iron clouds (e.g., Ackerman \& Marley 2001), whose condensate pressures are not far away from each other. Once a detached convective zone is formed by radiative cloud heating, the silicate and iron cloud layers are expected to evolve together.

Possibly more "blurred" cloud structures could occur, with particles comprising "dirty grains" comprising both silicates and iron, as suggested by detailed microphysical models by Helling's group, as discussed above. We argue that this chemical complexity would not suppress the variability. Even though the cloud structure in Helling's model could be smoother than the multilayer cloud models predicted by equilibrium chemistry, a sharp transition to nearly cloud-free will still occur near the base of the cloud layer (see figures in, e.g., Helling et al. 2008b), presumably due to evaporation of silicate and iron components. This is the same as our model, in which a sharp transition of the cloud base can result in a detached secondary convective zone. Meanwhile, the sharpness and location of the cloud top in our model are determined by interactions between convection and the cloud radiative effect but not by the composition of the clouds. Therefore, we expect that the sharp cloud top, and thus the variability, would still exist even if the cloud microphysics was treated similarly as in Helling's model.

\subsection{Implications for Global Atmospheric Circulation}

One of the key interests in atmospheric circulation of BDs and directly imaged EGPs is whether large-scale flows are dominated by zonally banded structure similar to Jupiter and Saturn. Classical two-dimensional turbulence theory predicts that banded structure can emerge from interactions between isotropic turbulence and the planetary rotation (see reviews by, e.g., Vasavada \& Showman 2005; Showman et al. 2018a). In the context of cloud-free BDs, radiation is usually thought of as a form of thermal damping, in that hot (cold) regions tend to radiatiate greater (lesser) flux to space, lessening the amplitude of thermal perturbations relative to the mean radiative equilibrium state (Showman \& Kaspi 2013; Zhang \& Showman 2014). If sufficiently strong, this radiative damping can damp out the atmospheric energy before it has time to reorganize into a zonally banded pattern comprising zonal jets (Zhang \& Showman 2014). Our study suggests, however, that the existence of clouds can modify this picture. In the presence of clouds, the local atmospheric temperature can undergo large variations over rapid timescales. The variation is unlikely to be globally isotropic but is nevertheless likely to be patchy on regional-to-large scales. Such patches may each experience cloud radiative feedback analogous to that captured in our 1D model but with different phasing of their evolution throughout the cloud cycle. This will both contribute to cloud patchiness that will help explain the observed IR light-curve variability and result in temperature differences on isobars up to hundreds of $\mathrm{K}$, depending on the cloud properties. As such, in addition to acting as a strong damping, cloud effects cause radiation to act as a patchy, potentially random forcing of the atmosphere. The size of a local patch that can be described by the 1D model is determined by large-scale dynamics in response to the multihundred $\mathrm{K}$ temperature perturbations. A natural dynamical length scale that might be relevant is the Rossby deformation radius, over which atmospheric motions are strongly affected by planetary rotation. Future 3D global models with cloud radiative feedback are necessary to investigate the large-scale dynamics that results from cloud feedback, as well as the selforganization of the surface patches.

The strong influence of radiative cloud feedback on the thermal structure raises an interesting question: how are the cloud effects coupled with the large-scale dynamics in BDs and directly imaged EGPs? In Earth's tropics, deep moist convection driven by condensational latent heating and the 
associated cloud formation strongly control the local temperature structure. Large-scale equatorial disturbances are triggered by and coupled with the moist convection, manifesting themselves as zonally propagating waves covering a broad range of the spectrum in both zonal wavenumber and frequency (Kiladis et al. 2009). These convectively coupled equatorial waves imprint rich and dominant signatures on the equatorial variability in the outgoing long-wave radiation (Wheeler \& Kiladis 1999). If the fundamental dynamical mechanisms driving the coupled equatorial waves work the same way in atmospheres of BDs and directly imaged EGPs, it is not surprising that similar waves triggered by cloud formation could exist in these atmospheres and be able to strongly affect the observed variability. Recently, Apai et al. (2017) showed that the puzzling evolution of long-term light curves of several BDs can be adequately explained by beating patterns caused by a pair of zonally propagating brightness disturbances that have different phase speeds, based on which they suggested the presence of banded structures similar to Neptune's atmosphere in these variable BDs. Here we imagine an alternative possible mechanism as the presence of coupled equatorial waves triggered by radiative cloud feedback. In this mechanism, the differential propagating brightness variation could be contributed by two dominant modes of the waves. Banded structure is not a necessity because the differential propagating waves can be clumped at the equator to produce the same observational signals as those from banded structures. Global atmospheric models are necessary to demonstrate the viability of these ideas. Also, retrieval models are needed to probe the temperature and cloud structures associated with brightness anomalies to test if this mechanism is at play.

\section{Conclusions}

Clouds remain one of the biggest obstacles in understanding ultracool atmospheres, including those of BDs and directly imaged EGPs. In particular, clouds are thought to be the major cause of observed light-curve variabilities of many BDs and directly imaged EGPs, as well as the long-standing, puzzling L-to-T dwarf transition. In this study, we have investigated the short-time evolution of clouds and thermal structures driven by radiative cloud feedback using a simple time-dependent 1D model that self-consistently treats cloud formation/dissipation, convective mixing, and radiative transfer. We conclude the following.

1. Radiative cloud feedback can drive spontaneous atmospheric variability in both temperature and cloud structure under conditions appropriate for BDs and directly imaged EGPs. The variability mainly comprises cycles in which clouds gradually dissipate after replenishment, and throughout the cycle, the cloud-base and cloud-top altitude, and thus the cloud thickness, vary over time. The atmospheric vertical temperature profile also evolves along with the cloud cycle. The typical periods of variability are 1 to tens of hr with a typical amplitude of the variability up to hundreds of $K$ in effective temperature. This is a novel, very natural mechanism to explain the observed variability in $\mathrm{L}$ and $\mathrm{T}$ dwarfs.

2. The mechanism responsible for the dissipation of clouds is simply the stratification below the cloud base due to cloud radiative heating, which suppresses the rapid supply of deep vapor and causes the net cloud sinks due to particle settling through the cloud base. After the cloud dissipates, the atmosphere near the cloud base cools off, which can either cause supersaturation of vapor or lessen the stratification (so that vapor can be mixed above the condensation level by convection), forming new clouds again. Over the cloud cycle, the cloud top grows to higher altitudes. The responsible mechanism is that the decreasing outgoing thermal flux due to the rising cloudtop altitude cools the atmosphere above the cloud top, and this cooling tends to drive a large temperature lapse rate just above the cloud top due to the large vertical gradient of the cloud mixing ratio near the cloud top, thus causing the instability to extend slightly higher in altitude. This then cools the above-cloud temperature profile even more, causing the cloud top to extend to even higher altitude. In our model, no artificial forcing, such as parameterized convective perturbations, is necessary for the variability to occur. It is a totally spontaneous, natural behavior of the system. This differs, for instance, from the radiative perturbations explored in the $1 \mathrm{D}$ model by Robinson \& Marley (2014), which had to be triggered by a perturbation imposed by hand deep in the domain.

3. The existence of variability is robust over a wide range of parameter space. However, the detailed evolution of variability is sensitive to model parameters. In general, the variability can be divided into regular or irregular types, the latter associated with the onset of chaos.

4. The radiative cloud-driven variability is appealing to explain the observed flux variability in BDs and some directly imaged EGPs, especially those evolving irregularly over short timescales. It is also a promising mechanism for cloud breaking, which has been proposed to explain the properties of the L-to-T dwarf transition.

5. A firm prediction is that thick clouds usually correlate with a convective and cooler profile, and thin clouds correlate to a stratified and warmer profile, which is a result of coevolution of clouds and thermal structure. This combination of surface types has been inferred for a few $\mathrm{BDs}$, and retrievals for more variable BDs in the future will help clarify whether this mechanism is prevalent among BDs.

We anticipate future endeavors in this direction. First, coupling of radiative cloud feedback into global atmospheric circulation models is essential to truly decipher the observed variability. Second, employing realistic radiative transfer and chemistry is key to understanding the spectroscopically resolved variability. Third, coupling with detailed cloud microphysics models will provide significant insights on the underlying mechanisms triggering the L-to-T transition.

We thank Tad Komacek, Vivien Parmentier, and Xi Zhang for helpful discussion. We thank the referee for comments that improve the manuscript. This work was supported by NASA Headquarters under the NASA Earth and Space Science Fellowship (Astrophysics) Program to X.T. and NSF grant AST 1313444 to A.P.S.

\section{Appendix A Vertical-resolution Test}

Here we show the vertical-resolution test of our model with $50,100,200$, and 300 vertical layers in the left panels of 

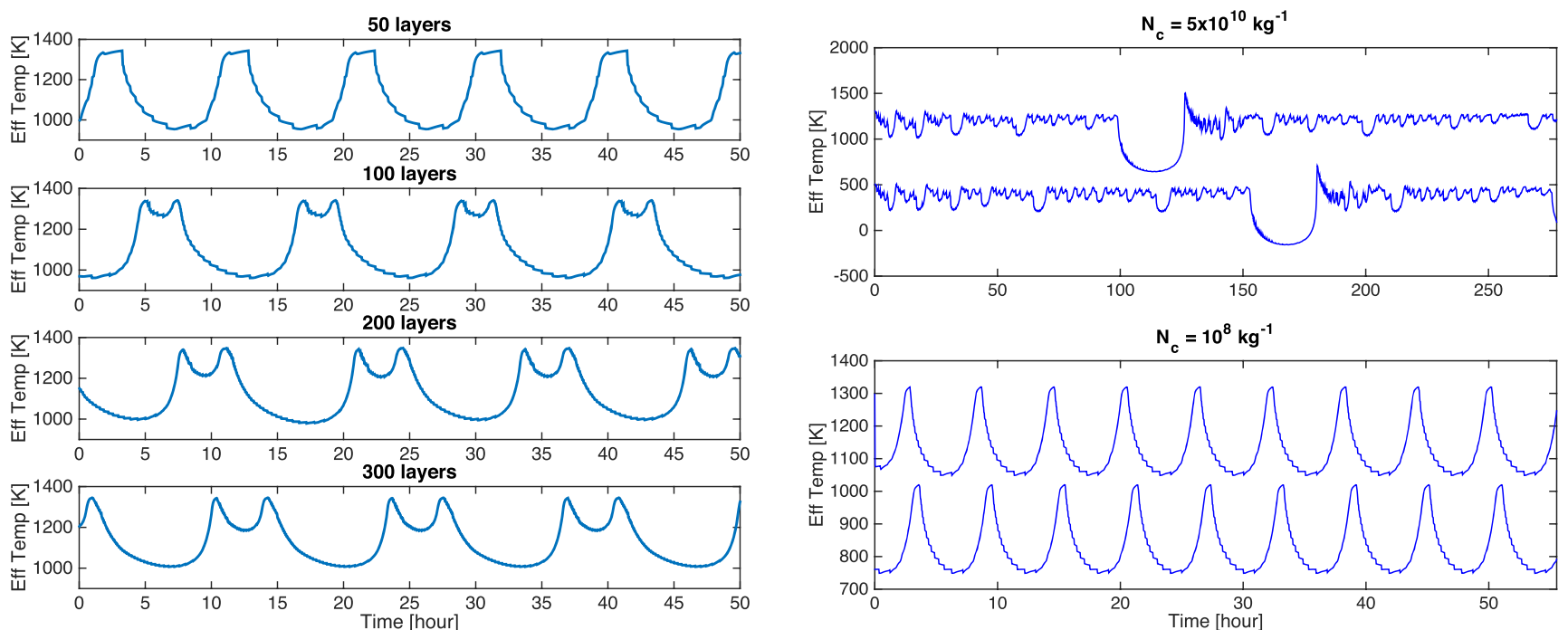

Figure 13. Left: outgoing thermal flux in terms of effective temperature as a function of time for cases with vertical resolution of 50, 100, 200, and 300 layers. Note that the 100-layer resolution is used in models presented in this work. The results show good agreement for models with 100 layers and more. Upper right panel: arbitrarily offset fluxes as a function of time for two cases with $\mathcal{N}_{c}=5 \times 10^{10} \mathrm{~kg}^{-1}$, in which the initial conditions are differed by only $1 \%$ of the cloud mixing ratio in one grid point. Lower right panel: fluxes for two cases with $\mathcal{N}_{c}=10^{8} \mathrm{~kg}^{-1}$, in which the first case initially has no cloud, and the second case has a thick cloud layer.

Figure 13, in which the 100-layer resolution is used for models presented in this work. Resolution much higher than 300 layers is too computationally expensive to integrate to a quasiequilibrium state primarily because of the extremely short diffusion timescale between adjacent model layers. The results show good agreement for models with 100 layers and more. As shown in the sensitivity studies, the detailed behavior of the variability is way more sensitive to physical assumptions of the model than resolutions higher than 100 layers. For the sake of understanding physical mechanisms, the 100-layer resolution is sufficient.

\section{Appendix B Sensitivity to Initial Conditions}

We perform sensitivity studies to initial conditions for the model with $\mathcal{N}_{c}=5 \times 10^{10} \mathrm{~kg}^{-1}$ and $\mathcal{N}_{c}=10^{8} \mathrm{~kg}^{-1}$. In the right panels of Figure 13, the upper panel shows evolution of fluxes that are offset arbitrarily for two cases in which the initial conditions differ by only $1 \%$ of the cloud mixing ratio in one grid point. It is easy to see that the evolution of the two models is sensitive to initial conditions. For models with $\mathcal{N}_{c}=10^{8} \mathrm{~kg}^{-1}$, shown in the lower panel, we test experiments with vastly different initial cloud structures, in which the first case initially has no cloud, and the second case has a thick cloud layer. One can see that the evolutions of fluxes quickly merge to periodic oscillations with almost the same frequency and amplitude, suggesting that this model is not sensitive to initial conditions.

\section{Appendix C Exponential Cloud Distribution}

As stated in Section 2.1, we have tested our model assuming an alternative cloud size-distribution function - the exponential distribution described by Equation (8). The variability in terms of effective temperature for models with different cloud number per mass $N_{c}$ is shown in Figure 14. Here we see the same trend as in Section 3.3.1, wherein smaller cloud number per mass (and hence larger cloud particles) results in regular
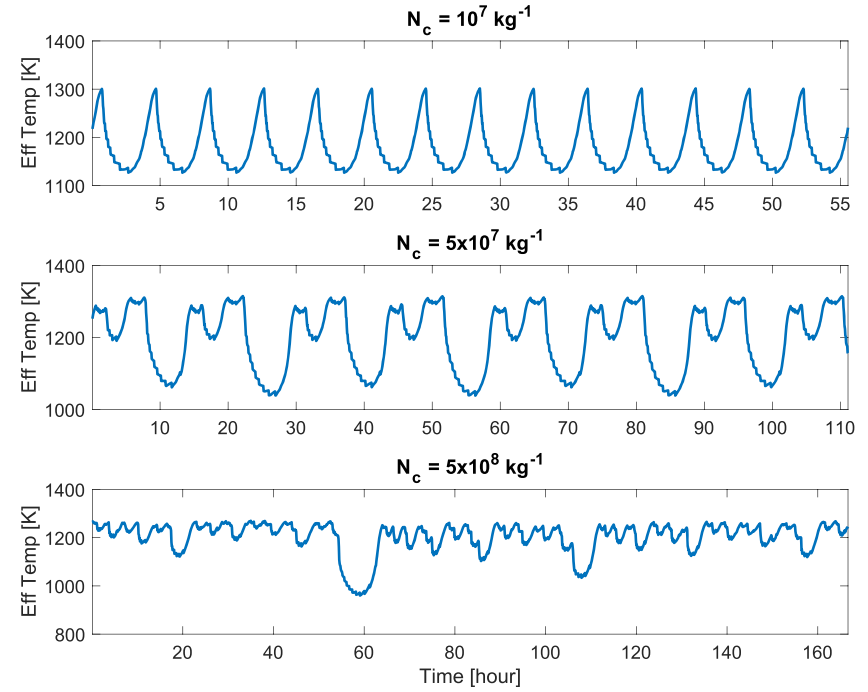

Figure 14. Effective temperature as a function of time for models assuming exponential cloud size distribution with three different cloud numbers per mass: $N_{c}=10^{7}, 5 \times 10^{7}$, and $5 \times 10^{8} \mathrm{~kg}^{-1}$.

and higher-frequency oscillations, whereas large cloud number per mass (small cloud particles) exhibits chaotic oscillations. In between, a model with mediate $N_{c}$ shows quasi-regular oscillations. Diagnoses similar to that in Section 3.1 show that the same physical mechanism is responsible for variability assuming exponential cloud size distribution.

\section{ORCID iDs}

Xianyu Tan (1) https://orcid.org/0000-0003-2278-6932

\section{References}

Ackerman, A. S., \& Marley, M. S. 2001, ApJ, 556, 872

Allard, F., Hauschildt, P. H., Alexander, D. R., Tamanai, A., \& Schweitzer, A. 2001, ApJ, 556, 357

Allard, F., Homeier, D., Freytag, B., \& Sharp, C. M. 2012, in Atmospheres From Very Low-Mass Stars to Extrasolar Planets, EAS Publications Ser. 57, ed. C. Reylé, C. Charbonnel, \& M. Schultheis (Paris: EDP Sciences), 3 
Apai, D., Karalidi, T., Marley, M., et al. 2017, Sci, 357, 683

Apai, D., Radigan, J., Buenzli, E., et al. 2013, ApJ, 768, 121

Artigau, É 2018, Variability of Brown Dwarfs, Handbook of Exoplanets (Berlin: Springer), 555

Artigau, É, Bouchard, S., Doyon, R., \& Lafrenière, D. 2009, ApJ, 701, 1534

Bailer-Jones, C. A. L., \& Mundt, R. 2001, A\&A, 367, 218

Barman, T. S., Macintosh, B., Konopacky, Q. M., \& Marois, C. 2011, ApJ, 733,65

Biller, B. 2017, AstRv, 13, 1

Biller, B. A., Crossfield, I. J. M., Mancini, L., et al. 2013, ApJL, 778, L10

Biller, B. A., Vos, J., Bonavita, M., et al. 2015, ApJL, 813, L23

Biller, B. A., Vos, J., Buenzli, E., et al. 2018, AJ, 155, 95

Buenzli, E., Apai, D., Morley, C. V., et al. 2012, ApJL, 760, L31

Buenzli, E., Apai, D., Radigan, J., Reid, I. N., \& Flateau, D. 2014, ApJ, 782, 77

Buenzli, E., Marley, M. S., Apai, D., et al. 2015, ApJ, 812, 163

Burgasser, A. J., Marley, M. S., Ackerman, A. S., et al. 2002, ApJL, 571, L151

Burningham, B., Marley, M. S., Line, M. R., et al. 2017, MNRAS, 470, 1177

Burrows, A., Sudarsky, D., \& Hubeny, I. 2006, ApJ, 640, 1063

Charnay, B., Bezard, B., Baudino, J.-L., et al. 2018, ApJ, 854, 172

Clarke, F. J., Hodgkin, S. T., Oppenheimer, B. R., Robertson, J., \& Haubois, X. 2008, MNRAS, 386, 2009

Cooper, C. S., Sudarsky, D., Milsom, J. A., Lunine, J. I., \& Burrows, A. 2003, ApJ, 586, 1320

Crossfield, I. J. M., Biller, B., Schlieder, J. E., et al. 2014, Natur, 505, 654

Durran, D. R. 1991, MWRv, 119, 702

Fletcher, N. H. 2011, The Physics of Rainclouds (Cambridge: Cambridge Univ. Press)

Freedman, R. S., Lustig-Yaeger, J., Fortney, J. J., et al. 2014, ApJS, 214, 25

Freytag, B., Allard, F., Ludwig, H.-G., Homeier, D., \& Steffen, M. 2010, A\&A, 513, A19

Gagné, J., Faherty, J. K., Burgasser, A. J., et al. 2017, ApJL, 841, L1

Gao, P., Marley, M. S., \& Ackerman, A. S. 2018, ApJ, 855, 86

Gao, P., Zhang, X., Crisp, D., Bardeen, C. G., \& Yung, Y. L. 2014, Icar, 231,83

Gelino, C. R., Marley, M. S., Holtzman, J. A., Ackerman, A. S., \& Lodders, K. 2002, ApJ, 577, 433

Gierasch, P., \& Goody, R. 1968, P\&SS, 16, 615

Gillon, M., Triaud, A., Jehin, E., et al. 2013, A\&A, 555, L5

Helling, C., Ackerman, A., Allard, F., et al. 2008b, MNRAS, 391, 1854

Helling, C., \& Casewell, S. 2014, A\&ARv, 22, 80

Helling, C., Dehn, M., Woitke, P., \& Hauschildt, P. H. 2008a, ApJL, 675, L105

Helling, C., \& Fomins, A. 2013, RSPTA, 371, 20110581

Helling, C., Oevermann, M., Lüttke, M., Klein, R., \& Sedlmayr, E. 2001, A\&A, 376, 194

Hiranaka, K., Cruz, K. L., Douglas, S. T., Marley, M. S., \& Baldassare, V. F. 2016, ApJ, 830, 96

Houze, R. A. 2014, Cloud Dynamics, Vol. 104 (New York: Academic)

Jäger, C., Dorschner, J., Mutschke, H., Posch, T., \& Henning, T. 2003, A\&A, 408, 193

Karalidi, T., Apai, D., Marley, M. S., \& Buenzli, E. 2016, ApJ, 825, 90
Kiladis, G. N., Wheeler, M. C., Haertel, P. T., Straub, K. H., \& Roundy, P. E. 2009, RvGeo, 47, RG2003

Kirkpatrick, J. D. 2005, ARA\&A, 43, 195

Komacek, T. D., Showman, A. P., \& Tan, X. 2017, ApJ, 835, 198

Kylling, A., Stamnes, K., \& Tsay, S.-C. 1995, JAtC, 21, 115

Lee, G., Dobbs-Dixon, I., Helling, C., Bognar, K., \& Woitke, P. 2016, A\&A, 594, A48

Leggett, S. K., Cushing, M. C., Hardegree-Ullman, K. K., et al. 2016, ApJ, 830,141

Line, M. R., Marley, M. S., Liu, M. C., et al. 2017, ApJ, 848, 83

Lines, S., Mayne, N., Boutle, I. A., et al. 2018, A\&A, 615, A97

Lodders, K. 2003, ApJ, 591, 1220

Lorenz, E. N. 1963, JAtS, 20, 130

Marley, M. S., \& Robinson, T. D. 2015, ARA\&A, 53, 279

Marley, M. S., Saumon, D., \& Goldblatt, C. 2010, ApJL, 723, L117

Metchev, S. A., Heinze, A., Apai, D., et al. 2015, ApJ, 799, 154

Miles-Páez, P. A., Pallé, E., \& Zapatero Osorio, M. R. 2017, MNRAS, 472, 2297

Morley, C. V., Fortney, J. J., Marley, M. S., et al. 2012, ApJ, 756, 172

Motter, A. E., \& Campbell, D. K. 2013, PhT, 66, 27

Parmentier, V., Showman, A. P., \& Lian, Y. 2013, A\&A, 558, A91

Powell, D., Zhang, X., Gao, P., \& Parmentier, V. 2018, ApJ, 860, 18

Pruppacher, H. R., \& Klett, J. D. 2012, Microphysics of Clouds and Precipitation: Reprinted 1980 (Berlin: Springer), 381

Radigan, J., Jayawardhana, R., Lafrenière, D., et al. 2012, ApJ, 750, 105

Radigan, J., Lafrenière, D., Jayawardhana, R., \& Artigau, E. 2014, ApJ, 793, 75

Rauscher, E., \& Menou, K. 2012, ApJ, 750, 96

Robinson, T. D., \& Marley, M. S. 2014, ApJ, 785, 158

Saumon, D., \& Marley, M. S. 2008, ApJ, 689, 1327

Schäfer, J., Lee, S.-C., \& Kienle, A. 2012, JQSRT, 113, 2113

Showman, A., Ingersoll, A., Achterberg, R., \& Kaspi, Y. 2018a, in The Global Atmospheric Circulation of Saturn, Saturn in the 21st Century, ed. K. Baines \& M. Flasar (Cambridge: Cambridge Univ. Press)

Showman, A. P., \& Kaspi, Y. 2013, ApJ, 776, 85

Showman, A. P., Tan, X., \& Zhang, X. 2018b, arXiv:1807.08433

Smith, M. D., \& Gierasch, P. J. 1995, Icar, 116, 159

Stephens, D. C., Leggett, S. K., Cushing, M. C., et al. 2009, ApJ, 702, 154

Straka, J. M. 2009, Cloud and Precipitation Microphysics: Principles and Parameterizations (Cambridge: Cambridge Univ.Press)

Tan, X., \& Showman, A. P. 2017, ApJ, 835, 186

Tsuji, T. 2002, ApJ, 575, 264

Vasavada, A. R., \& Showman, A. P. 2005, RPPh, 68, 1935

Visscher, C., Lodders, K., \& Fegley, B., Jr 2006, ApJ, 648, 1181

Visscher, C., Lodders, K., \& Fegley, B., Jr. 2010, ApJ, 716, 1060

Wheeler, M., \& Kiladis, G. N. 1999, JAtS, 56, 374

Wilson, P. A., Rajan, A., \& Patience, J. 2014, A\&A, 566, A111

Woitke, P., \& Helling, C. 2004, A\&A, 414, 335

Yang, H., Apai, D., Marley, M. S., et al. 2016, ApJ, 826, 8

Zhang, X., \& Showman, A. P. 2014, ApJL, 788, L6

Zhou, Y., Apai, D., Schneider, G. H., Marley, M. S., \& Showman, A. P. 2016, ApJ, 818, 176 